\title{
Electronic-controlled Tensile Tester
}

\author{
How the Control System is Designed; \\ Some Test Results Given.
}

By Takashi Murakami and Shigeru Aonuma

Faculty of Textile, Shinshu University, Ueda, Japan

Based on Journal of the Textile Machinery Society of Japan, Transactions, Vol. 18, No. 10, T 632-641 (1965) and Vol. 19, No. 3, T73-84 (1966)

\begin{abstract}
Here is an all-purpose electronic tensile tester experimentally produced. It can impose loads or extensions linearly or nonlinearly on test pieces by its automatic control mechanism. The mechanism has an analog computer which serves as a program generator. The characteristics of the tester, when used for tensile tests, and the results of the tests are :

(1) This tester is made up of three parts ; i) a program generator which converts an arbitrary load or extension into an input voltage; ii) a controller of input loads; and iii) an output detector-recorder.

(2) The overall transfer function of the tester under load input is:

$$
G_{o f}(s)=\begin{gathered}
K_{A} G_{p}(s) \\
s\left(T_{M} s-1\right)
\end{gathered} e^{-L S}
$$
\end{abstract}

where $G_{p}(s)$ is the transfer function of a test piece. If a spring is placed where a test piece would be placed, the frequency response of the open loop is stable at $\omega 45$ $\mathrm{rad} / \mathrm{sec}$ or less, even if the gain is $45 \mathrm{~dB}$.

(3) The overall transfer function of the tester under extension input is as shown below, the closed-loop fre uency characteristic closely resembling that in a case where a spring is interposed under load input:

$$
G_{o x}(s) \quad s\left(T_{M} S \cdot 1\right) e^{e L S}
$$

(4) The closed-loop transfer function under load input is :

$$
G_{f}(s)=\begin{array}{c:c}
K_{c} \\
T_{M} s^{2} & 1 \quad K_{A} L G_{p}(s) ! s: K_{A} G_{p}(s)
\end{array} e^{-L s}
$$

If a spring is placed where a test piece would be placed, the closed-loop fre uency is stable at (1) $30 \mathrm{rad} / \mathrm{sec}$ or less, even if the gain is $32.5 \mathrm{~dB}$.

(5) The closed-loop transfer function under deformation input is shown by this e`uation :

$$
G_{x}(s)=T_{M} s^{2} \cdot\left(1-K_{B} L\right) s: K_{B} e^{-L S}
$$

With a spring used as a test piece, the closed loop frequency resembles that in a case where a spring is used as a test piece under load input.

(6) This tester has been experimentally proved capable of testing repeated tensile testing hitherto difficult. It is also capable of tests under loads or deformation input in various functions, e.g., tests under constant-speed load input.

(7) The mechanical transient characteristic of gum and fibers, ascertained from the results of tests under loads and deformation input, has the same tendency as a theoretical curve. This attests to the fidelity of the tester.

(8) Tests by our tester of the frequency response of fibers under loads and deformation input have shown that the phase leads if deformation input is used. By "phase leads" we mean "phase shifts to positive."

(9) The load output of the transfer function of Voigt-Maxwell's four-element model always shows in the function of $\sinh x(t)$, if the input is of deformation type.

(10) Some applied examples of tests possible by this machine will be given.

We believe from the evidence that this tester is eminently suited for testing the dynamic mechanical properties of general industrial materials. 


\section{Introduction}

Roughly, there are two methods to test industrial materials, e.g., fibers, rubber and plastics. One method is the load-input type; the other, the deformation (extension) input type.

The conventional machines for testing industrial materials, including all-purpose machines, such as "Instron," are mostly of a constant-speed deformation input type. Machines of load input type, such as the in cline method and the wedge-balance load method of a constant-speed loading type, are subject to the influence of the mass of their movable parts, or contain nonlinear elements.

To investigate the characteristics of materials under various mechanical conditions depending on the purpose of tests, we need a tester which can impose a variety of loading or deformation. We have experimentally manufactured an all-purpose electronic tensile tester of an automatic-balance type which uses electron tubes and an analog computer as a program generator and is capable of tests under various program functions.

Let us show how to use this tester for tensile tests, describe its characteristics, discuss the results of tests and give a few examples of the tests.

\section{Experimental Production of Tensile Tester}

2-1. The Tester- How to Use it

This tester is made up of three parts: i) the program generator which converts loads or extensions imposed on the test piece into input voltages according to a prescribed program function; ii) the load input controller which transfers load input faithfully; and iii) the detector and recorder of output (load or deformation).

\section{2-1-1. Load input}

Fig. 1 is a diagram of the tester where it is used with a load as input. The load to be imposed on

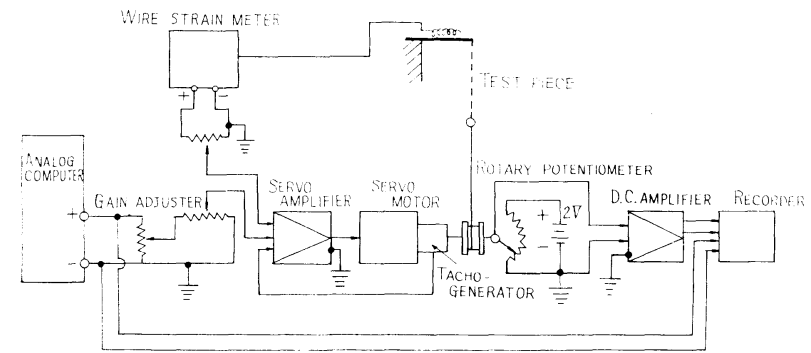

Fig. 1 Electronic tensile tester (load input type) the test piece is pre-programmed and converted into an input voltage. It is supplied to the servo amplifier through the gain adjuster and converted into an extension by driving the servo-motor. Thus the prescribed load can be to put on the test piece.

The load, after conversion into voltage by the upper terminal which detects the load, is compared with the input voltage. Then the terminal of displacement is driven until the deflection voltage is zero. Thus we can impose the load on the test piece faithfully according to the program function.

The deformation (extension or compression) of the test piece is converted into voltage by the rotary potentiometer coupled with the servo-mator. It is then sent to the recorder through the D.C. amplifier for recording together with the input signal.

2-1-2. Extension input

To make a test when input is of the extension type, rearrange the machine as shown by Fig. 2. As in the case of load input, the extension to be put on the test piece should be programmed in the analog computer and converted into an input voltage. Then the input voltage powers the servo-motor through the gain-adjuster and servo-amplifier, and gives extension to the test piece.

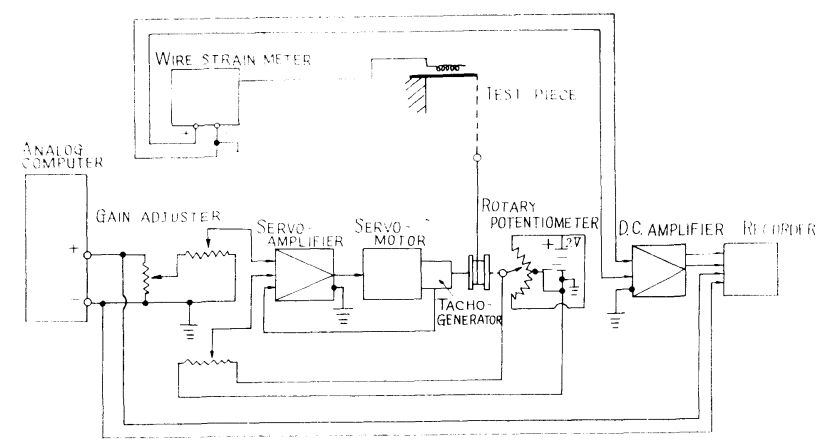

Fig. 2 Electronic tensile tester (extension input type)

The rotation angles of the servo-motor proportional to this extension is converted into voltage by the rotary potentiometer. The voltage is then compared with the previous input voltage and powers the servomotor until the deflection voltage is zero. Thus the input extension is loaded faithfully on the test piece. The force which generates from the test piece is detected by the upper detecting terminal and records itself on the recorder via the D.C. amplifier.

2-2. Characteristics of Tester

2-2-1. Load input

Fig. 3 is a block diagram of the Tester when it uses a load as input, as in Fig. 1, where: 
$f(t)$ : input load

$x(t)$ : output displacement

$K_{1}$ : gain constant of program generator

$K_{2}$ : gain constant of servo-amplifier

$K_{3} /\left(T_{M}{ }^{\prime} s+1\right):$ transfer function of servo-motor

$T_{M^{\prime}}$ : time constant of servo-motor

$1 / s:$ transfer function of position

$e^{-L S}:$ transfer function of time-lag of servo-motor

$r$ : gain constant for converting the rotary angle of servo-motor into displacement

$G_{p}(s)$ : transfer function of test piece

$K_{4}$ : gain constant of wire strain meter

$K_{5}$ : gain constant of rotary potentiometer

$K_{6}$ : gain constant of D. C. amplifier

$K_{7}$ : gain constant of recorder

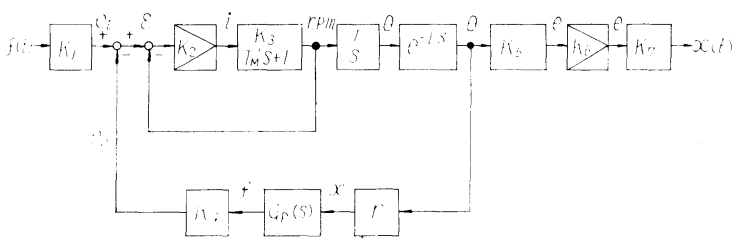

Fig. 3 Block diagram of load input tensile tester

Assume that the transfer function of the minorloop - which includes the servo-amplifier and the servomotor-is $G_{M}(s)=K_{M} /\left(T_{M} s+1\right)$. Then we obtain from Fig. 3 the overall transfer function of the tester, as follows :

$$
G_{o f}(s)=\frac{K_{A} G_{p}(s)}{s\left(T_{M} s+1\right)} e^{L S}
$$

where

$$
K_{A}=K_{M} K_{4} r, \quad K_{M}=\begin{gathered}
K_{2} K_{3} \\
1+K_{2} K_{3}
\end{gathered}, \quad T_{M}=\begin{gathered}
T_{M^{\prime}} \\
1+K_{2} K_{3}
\end{gathered}
$$

Therfore, the open-loop frequency response of this tester under load input is :

$$
\begin{aligned}
\left|G_{o f}\right| & =\frac{K_{A}\left|G_{p}(j \omega)\right|}{\omega \sqrt{ } 1+\left(T_{M} \omega\right)^{2}} \\
N \mathrm{~dB} & =20 \log _{10} K_{A}+20 \log _{10}\left|G_{p}(j \omega)\right|-20 \log _{10 \omega} \\
& -10 \log _{10}\left\{1+\left(T_{M} \omega\right)^{2}\right\} \\
\phi= & \angle G_{p}(j \omega)-\angle 90^{\circ}-\tan ^{-1} T_{M} \omega-\angle L \omega \cdots \cdots \cdots
\end{aligned}
$$

Fig. 4 shows a Bode diagram obtained by substituting the transfer function of a spring into $G_{p}(s)$ and by assuming $K_{S}=K_{A}=1, T_{M}=0.029 \mathrm{sec}$ and $L=0.014$ sec, where $T_{M}$ and $L$ are the values actually measured.

The solid line in Fig. 4 shows curves obtained by calculation. The marks plotted along the phase curve are the values actually measured. The measured values almost agree with the calculated values. If we raise the gain by $35 \mathrm{~dB}$, this tester is stable at $\omega=$
$45 \mathrm{rad} / \mathrm{sec}$ (about $7 \mathrm{c} / \mathrm{sec}$ ) or less. It is sufficient, then, to adjust the gain properly.

The usual method of theoretical analysis of the results of tests of materials uses force as input. Therefore, we analyze the closed-loop frequency response where load input is used. Closed-loop transfer function $G_{f}(s)$ is obtainable as follows from Fig. 3 :

$$
G_{f}(s)=\frac{K_{c}}{T_{M} s^{2}+\left\{1-K_{A} L G_{p}(s)\right\} s+K_{A} G_{p}^{-}(s)^{-e^{-L s}}}
$$

where

$$
K_{c}=K_{1} K_{5} K_{6} K_{7} K_{M}
$$

The terms of the second and subsequent orders of the expansion of $e^{-L S}$ being extremely small, we approximate $e^{-L S}$ to $e^{-L S} \doteqdot 1-L s$. Therefore, the transfer function and frequency characteristic differ depending on the contents of $G_{p}(s)$.

Substituting spring $\left(K_{s}\right)$ into $G_{p}(s)$ of eq. (5) re sults in the following equation:

$$
G_{s f}(s)=\frac{K_{c}}{T_{M} s^{2}+\left(1-K_{A} K_{s} L\right) s+K_{A} K_{s}{ }^{L s} \cdots(6)}
$$

where $G_{s f}(s)$ is the closed-loop transfer function when an ideal spring is put into the tester under load input. Accordingly, the frequency response is :

$$
\begin{aligned}
\left|G_{s f}\right| & =\sqrt{ }\left(K_{A} K_{S}-T_{M} \omega^{2}\right)^{2}+\left(1-K_{A} K_{S} L\right)^{2} \omega^{2} \\
\cdots \cdots \cdots(7) & \\
N \mathrm{~dB} & =20 \log _{10} K_{C}-10 \log _{10}\left\{\left(K_{A} K_{S}-T_{M} \omega^{2}\right)^{2}\right. \\
& \left.+\left(1-K_{A} K_{S} L\right)^{2} \omega^{2}\right\}
\end{aligned}
$$

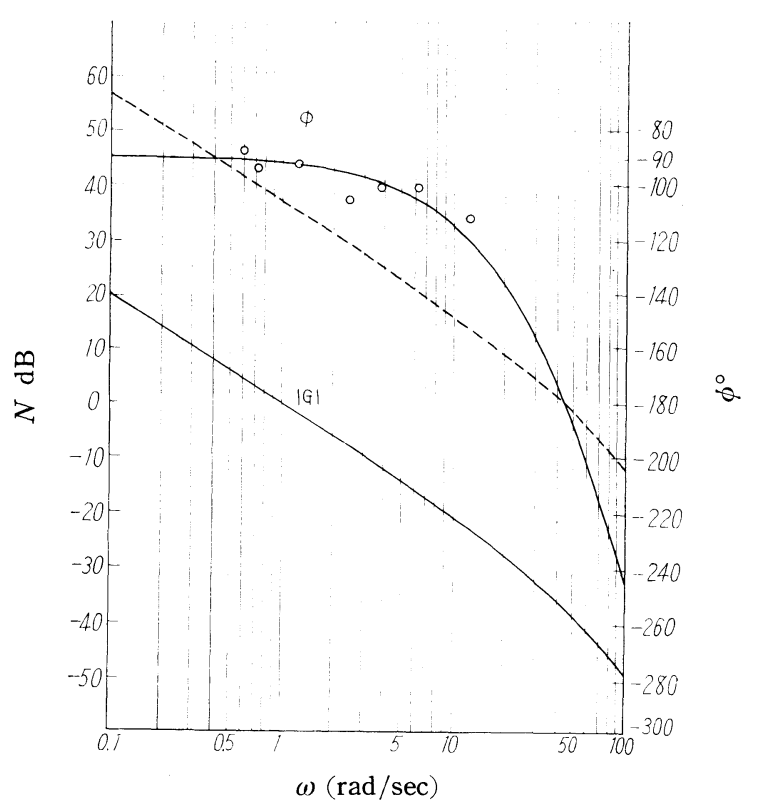

Fig. 4 Bode diagram (open-loop) of load input tensile tester 


$$
\phi=-\tan ^{-1}-\frac{\left(1-K_{A} K_{s} L\right) \omega}{K_{A} K_{s}-T_{M} \omega^{2}}-\angle L \omega
$$

where $\phi=0^{\circ}$ as $\omega=0$

and $\phi=-\infty^{\circ}$ as $\omega=\infty$

Fig. 5 is a closed-loop Bode diagram obtained by using a spring as a test piece and assuming $T_{M}=0.029$ sec, $L=0.014 \mathrm{sec}$ (an in Fig. 4) and $K_{c}=K_{A}=K_{s}=1$.

The marks plotted in this figure are measured values obtained by putting a spring into $G_{p}(s)$. The calculated values almost agree with the measured values. The dotted line in this figure is drawn by parallel transfer of the gain curve to the phase cross. over. If we raise the gain by $32.5 \mathrm{~dB}$, this system is stable at $\omega=30 \mathrm{rad} / \mathrm{sec}$ or less.

Fig. 6 is an example of frequency responses curves memorized on the storage oscilograph.

\section{2-2-2. Extension input}

Fig. 7 is a block diagram of the extension input tester shown in Fig. 2. Its overall transfer function is obtainable from this figure as follows:

$$
G_{o x}(s)=\frac{K_{B}}{s\left(T_{M} s+1\right)} e^{-L S}
$$

where

$$
K_{B}=K_{M} K_{5}
$$

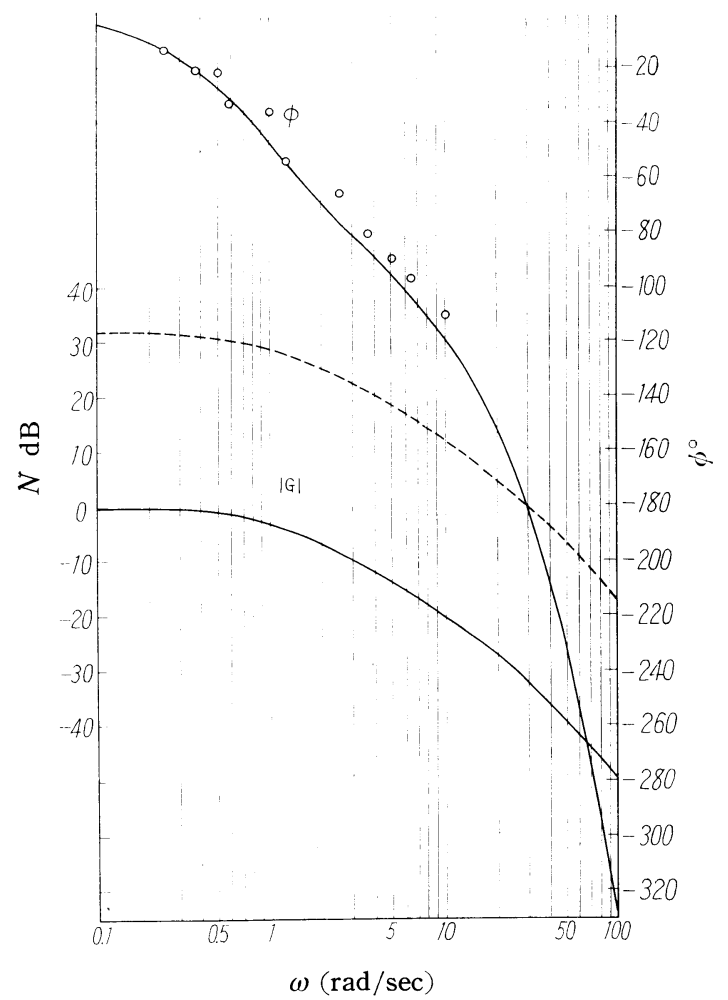

Fig. 5 Bode diagram of feed back system for load input tensile tester tested on spring
Inasmuch as the overall transfer function, in this case, does not include $G_{p}(s)$, the characteristic is of the tester alone. Hence the open-loop frequency response of the tester under extension input is :

$$
\begin{aligned}
\left|G_{o x}\right| & =\frac{K_{B}}{\omega \sqrt{1+\left(T_{M \omega)^{2}}\right.}} \\
N \mathrm{~dB} & =20 \log _{10} K_{B}-20 \log _{10} \omega-10 \log _{10}\left\{1+\left(T_{M \omega}\right)^{2}\right\}
\end{aligned}
$$

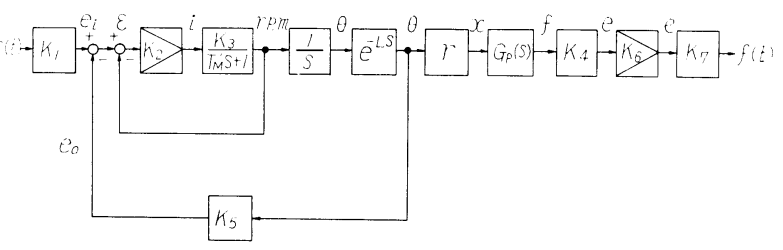

Fig. 7 Block diagram of extension input tensile tester

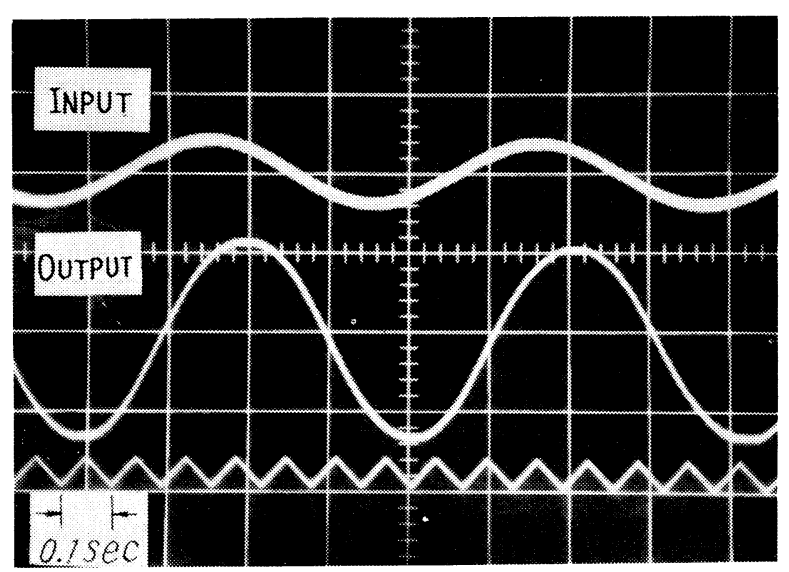

a) $\omega=0.96 \mathrm{rad} / \mathrm{sec}$

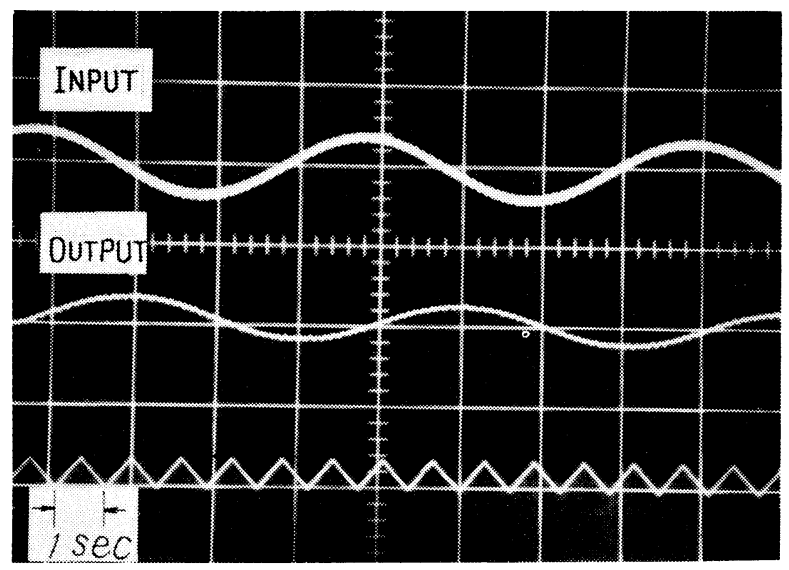

b) $\omega=9.6 \mathrm{rad} / \mathrm{sec}$

Fig. 6 Frequency response curve (load input type) 


$$
\phi=-\angle 90^{\circ}-\tan ^{-1} T_{M \omega}-\angle L \omega
$$

where $\phi=-90^{\circ}$ as $\omega=0$ and $\phi=-\infty^{\circ}$ as $\omega=\infty$

A Bode diagram drawn by assuming $K_{B}=1$ is omitted here because it closely resembles Fig. 4 . Therefore, the open-loop frequency response where extension input is used is the same as that where load input is used with a spring inserted.

The closed-loop frequency characteristic of this tester when extension input is used as on the conventional tester, is analyzable as shown below. As is clear from Fig. 7, the transfer function $G_{p}(s)$ of a test piece has no direct relation to the feed back loop. Therefore, let us look for closed-loop transfer function $G_{A x}(s)$ and frequency response of only the tester, they are :

$$
G_{A x}(s)=\frac{K_{E}}{T_{M} s^{2}+\left(1-K_{B} L\right) s+K_{B}^{-L S}} \cdots \cdots \cdots(14)
$$

where

$$
\begin{aligned}
& K_{E}=K_{1} K_{M}, K_{B}=K_{M} K_{5} \\
& \left.G_{A x}\right)=\sqrt{ }\left(K_{B}-T_{M} \omega^{2}\right)^{2}+\left(1-K_{B} L\right)^{2} \omega^{2} \\
& \begin{aligned}
N \mathrm{~dB} & =20 \log _{10} K_{E}-10 \log _{10}\left\{\left(K_{B}-T_{M} \omega^{2}\right)^{2}\right. \\
& \left.\quad+\left(1-K_{B} L\right)^{2} \omega^{2}\right\}
\end{aligned} \\
& \phi_{1}=-\tan ^{-1}\left(1-K_{B} L\right) \omega \\
& \phi=0^{\circ} \text { as } \omega=0 \\
& \phi=-T_{M} \text { as } \omega=\infty
\end{aligned}
$$

The transfer function $G_{x}(s)$ and the frequency response of the whole system, including $G_{p}(s)$, is :

$$
G_{x}(s)=\begin{gathered}
K_{H} G_{p}(s) \\
\left.T_{M} s^{2}-\left(1-K_{B} L\right) s+K_{B} e^{-L s} \quad \cdots \cdots \cdots \cdot 18\right)
\end{gathered}
$$

where

$$
\begin{aligned}
& K_{H}=K_{E} K_{4} K_{6} K_{7} r \\
& \left.G_{x} \mid=\frac{K_{H} \mid G_{p}(j \omega)}{\sqrt{ }\left(K_{B}-T_{M} \omega^{2}\right)^{2}+\left(1-K_{B} L\right)^{2} \omega^{2}} \cdots \cdots \cdots \cdot 19\right) \\
& N \mathrm{~dB}=20 \log _{10} K_{H}+20 \log _{10} G_{p}(j \omega)-10 \log _{10} \\
& \left.\quad\left\{\left(K_{B}-T_{M} \omega^{2}\right)^{2}+\left(1-K_{B} L\right)^{2} \omega^{2}\right\} \cdots \cdots \cdots \cdot 20\right\} \\
& \left.\phi_{2}=\angle G_{p}(j \omega)-\tan ^{-1} \frac{\left(1-K_{B} L\right) \omega}{K_{B}-T_{M} \omega^{2}}-\angle L \omega \cdots \cdots \cdot 21\right)
\end{aligned}
$$

Therefore, the characteristic of $G_{p}(s)$ of a test piece alone is obtainable by subtracting the frequency characteristic of the tester from that of the whole system. Exactly, it is obtainable by comparing the final output of the tester with the input for frequency response picked up from the output terminal of the feed back loop in Fig. 7. A further analysis which takes into consideration the contents of $G_{p}(s)$ is called for and will be given on a future occasion.

\section{Dynamic Characteristic of Test Pieces}

It is difficult, with the conventional tensile testers, to test a constant-speed load input or repeated stressing. With our tester it is possible to make tests under the input of loads or extensions of an arbitrary function generated from the function generator which uses an analog electronic computer.

We have made a wide variety of conceivable practical tests of tensile strength. However, materials made of highly polymerized compounds, rather than, say, iron which is limited in viscosity, are better suited as test pieces for demonstrating the efficiency of our tester and the effect of viscosity. Therefore, we give examples of tests on gum and fibers.

Advanced research is being made in the field of rheology as to the mechanical analysis of visco-elastic materials and their linear and non linear behavior. However, the purpose of this article being to discuss the efficiency of our tester, we will deal with viscoelastic materials by their transfer function, which is the usual technique in control engineering, and analyze the results of load and extension input tests, with special reference to the linear areas of such materials. 3-1. Analysis of Load Input Test

Substituting the transfer function of a fiber model into $G_{p}(s)$ of eq. (5), we obtain its dynamic characteristic. It is common knowledge, however, that mechanical properties of fibers are represented by VoigtMaxwell's four-element model. Accordingly, we will obtain the transfer function of the fiber model approximately by thinking of it as a four-element model.

3-1-1. Transient characteristic

A block diagram of our tester (Fig. 3) shows clearly that the transfer function $G_{p}(s)$ of a test piece has extension as input and force as output if load input is used. The ransfer function of the test piece is, therefore :

where

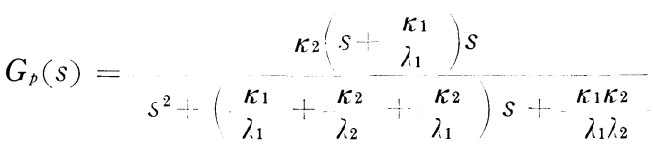

$\lambda_{1}, \lambda_{2}$ : damper coefficient of fiber model $(\mathrm{kg} \mathrm{sec} / \mathrm{cm})$

$\kappa_{1}, \kappa_{2}:$ spring constant $(\mathrm{Kg} / \mathrm{cm})$

Assuming $\kappa_{1}=\kappa_{2}=\kappa, \lambda_{1}=\lambda_{2}=\lambda, \lambda / \kappa=T$. and substituting them into eq. (5), we obtain the closed-loop transfer function $G_{V M f}(s)$ of the load input tester with a fiber model put into it:

$$
\left.G_{V M f}(s)=\frac{A s^{2}+B s+C}{s\left(a_{2} s^{3}+b_{2} s^{2}+c_{2} s+d_{2}\right)} e^{-L s} \quad \cdots \cdots \cdots \cdot 23\right)
$$

where

$$
\begin{aligned}
& a_{2}=T T_{M} \\
& b_{2}=T+3 T_{M}-\kappa K_{D} L T
\end{aligned}
$$




$$
\begin{aligned}
& c_{2}=3+T_{M} / T-\kappa K_{D}(L-T) \\
& A=K_{c} T \\
& B=3 K_{c} \\
& C=K_{c} / T
\end{aligned}
$$

Substituting measured values into $T_{M}, L$ and $T$ in this formula, we obtain the solutions, by an analog computer, to load input of various types: (a) step, (b) constant-speed and (c) constant-acceleration, as shown in Fig. 8.
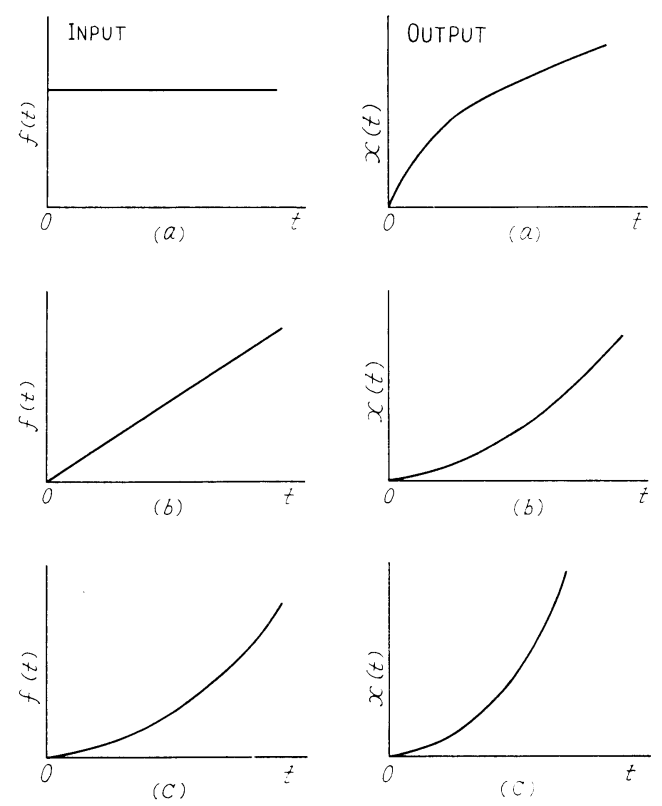

Fig. 8 Analog Computer Analysis of input load tester with fiber model inserted

The following equations, obtained by using the method Murakami[1] applied to constant-speed load output, show the responses of output displacement corres ponding to various types of load input of the fiber model. Fig. 9 gives output displacement responses corresponding to these types of load input.

(a) Where $f(t)=:(t)$

The output displacement response corresponding to step-type load input, shown in Fig. 9 (a), makes the creep curve expressed by the following equation :

$$
x(t)=\frac{1}{\kappa_{1}}\left(1-e-i_{1}^{1} t\right)+\frac{1}{\lambda_{2}} t+\frac{1}{\kappa 2}
$$

where $x(0)=1 / \kappa^{2}$ as $t=0$

and $\quad x(\infty)=\infty$ as $t=\infty$

(b) Where $f(t)=a t$

The output displacement response corresponding to constant-speed load input shown in Fig. 9 (b) is :
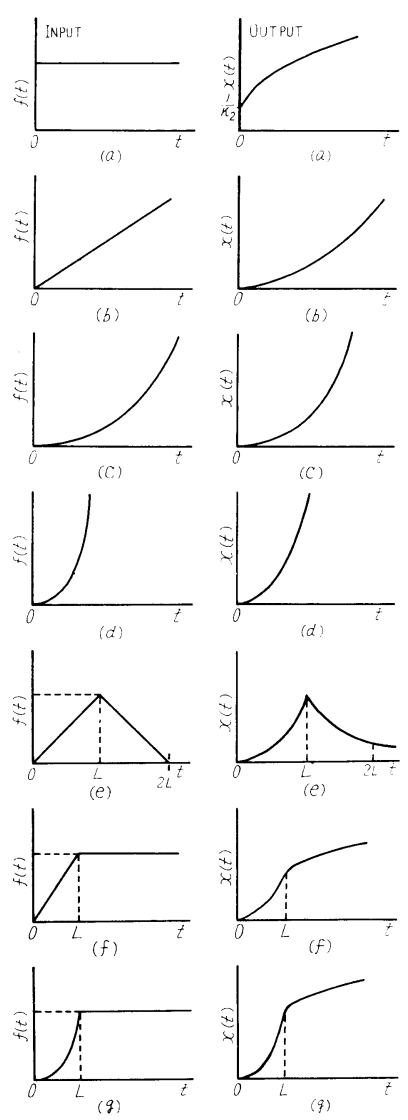

Fig. 9 Output extension response curve for several types of input load

$$
x(t)=a\left\{\frac{1}{2 \lambda_{2}} t^{2}+\left(\frac{1}{\kappa_{1}}+\frac{1}{\kappa_{2}} t-\frac{\lambda_{1}}{\kappa_{1}{ }^{2}}\left(1-e^{-\frac{\kappa_{1}}{\lambda_{1}} t}\right)\right\}\right.
$$

where $x(0)=0$ as $t=0$

and $\quad x(\infty)=\infty$ as $\mathrm{t}=\infty$

(c) Where $f(t)=\frac{a}{2} t^{2}$

The output displacement response correspoding to the constant-accelaration load input shown in Fig. 9 (c) is :

$$
\begin{aligned}
x(t) & =a\left\{\begin{array}{l}
1 \\
6\rangle_{2}
\end{array} t^{3}+\frac{1}{2}\left(\frac{1}{\kappa_{1}}+\frac{1}{\kappa_{2}} t^{2}-\frac{\lambda_{1}}{\kappa_{1}{ }^{2}} t\right.\right. \\
& +\frac{\lambda_{1}{ }^{2}}{\kappa_{1}{ }^{3}}\left(1-e^{-\frac{\kappa_{1}}{\lambda_{1}}} t\right)
\end{aligned}
$$

where $x(0)=0$ as $t=0$

and $\quad x(\infty)=\infty$ as $t=\infty$

(d) Where $f(t)=\frac{a}{6} t^{3}$

The output displacement response corresponding 
to the high-speed (impact) load input shown in Fig. 9 (d) is :

$$
\begin{aligned}
x(t) & =a\left\{\frac{1}{\left(24 \lambda_{2}\right.} t^{4}+\frac{1}{6}\left(\frac{1}{\kappa_{1}}+\frac{1}{\kappa_{2}}\right) t^{3}-\frac{\lambda_{1}}{2 \kappa_{1}{ }^{2}} t^{2}\right. \\
& \left.+\frac{\lambda_{1}{ }^{2}}{\kappa_{1}{ }^{3}} t-\frac{\lambda_{1}{ }^{3}}{\kappa_{1}{ }^{4}}\left(1-e^{-\frac{\kappa_{1}}{\lambda_{1}} t}\right)\right\}
\end{aligned}
$$

where $\quad x(0)=0 \quad$ as $t=0$

and $\quad x(\infty)=\infty$ as $t=\infty$

We can obtain, in the same way, output displacement responses corresponding to a triangular wave and combined load input :

(e) Where

$$
f(t)=a\{t-2(t-L) u(t-L)+(t-2 L) u(t-2 L)\}
$$

The output displacement response corresponding to the triangular-wave load input shown in Fig. 9 (e) is:

$$
\begin{aligned}
x(t) & =a_{L}^{\ulcorner} \frac{1}{2 \lambda_{2}} t^{2}-\frac{1}{\lambda_{2}}\left\{(t-L)^{2}-\frac{1}{2}(t-2 L)^{2}\right\} \\
& +\left(\frac{1}{\kappa_{1}}+\frac{1}{\kappa_{2}}\right) t-2\left(\frac{1}{\kappa_{1}}+\frac{1}{\kappa_{2}}\right)(t-L) \\
& +\left(\frac{1}{\kappa_{1}}+\frac{1}{\kappa_{2}}\right)(t-2 L)+\frac{\lambda_{1}}{\kappa_{1}}{ }^{2}\left\{e^{-\frac{\kappa_{1}}{\lambda_{1}} t}\right. \\
& -2 e^{-\frac{\kappa_{1}}{\lambda_{1}}(t-L)}+e^{-\frac{\kappa_{1}}{\lambda_{1}}(t-2 L)}-1+2 u(t-L) \\
& -u(t-2 L)\}]
\end{aligned}
$$

where $x(0)=0 \quad$ as $t=0$

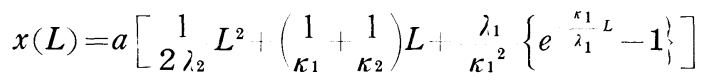$$
\text { as } t=L
$$

and $\quad x(2 L)=a\left\{\frac{1}{\lambda_{2}} L^{2}+\frac{\lambda_{1}}{\kappa_{1}{ }^{2}}\left(e^{-\frac{2 \kappa_{1}}{\lambda_{1}} L}-2 e^{-\frac{\kappa_{1}}{\lambda_{1}} L}+1\right)\right\}$

$$
\text { as } t=2 L \text {. }
$$

(f) Where $f(t)=a t\{1-u(t-L)\}+c u(t-L)$

The output displacement response corresponding to the combined-load input shown in Fig. 9 (f) is :

$$
\begin{array}{r}
x(t)=a_{L 2 \lambda_{2}}^{\ulcorner} \frac{1}{{ }^{2}}\left\{t^{2}-(t-L)^{2}\right\}+\left(\frac{1}{\kappa_{1}}+\frac{1}{\kappa_{2}}\right)\{t-(t-L)\} \\
\left.+\frac{\lambda_{1}}{\kappa_{1}{ }^{2}}\left\{e^{-\frac{\kappa_{1}}{\lambda_{1}}}-e^{-\frac{\kappa_{1}}{\lambda^{1}} t-L}+\mathcal{U}(t-L)-1\right\}\right] \cdots(29)
\end{array}
$$

where $x(0)=0 \quad$ as $t=0$

$$
\begin{aligned}
x(L)= & a\left\{\frac{L^{2}}{2 \lambda_{2}}+\left(\frac{1}{\kappa_{1}}+\frac{1}{\kappa_{2}}\right) L+\frac{\lambda_{1}}{\kappa_{1}{ }^{2}}\left(e^{-\frac{\kappa_{1}}{\lambda_{1}} L}-1\right)\right\} \\
& \text { as } t=L
\end{aligned}
$$

and $\quad x(\infty)=\infty \quad$ as $t=\infty$

(g) Where $f(t)=\frac{1}{2} a t^{2}\{1-u(t-L)\}+c u(t-L)$

The output displacement response corresponding to the combined-load input shown in Fig. $9(\mathrm{~g})$ is :

$$
\begin{aligned}
x(t) & =a_{L}^{\ulcorner} \frac{1}{6 \lambda_{2}}\left\{t^{3}-(t-L)^{3}\right\}+\frac{1}{2}\left(\frac{1}{\kappa_{1}}+\frac{1}{\kappa_{2}}\right) t^{2} \\
& -\frac{1}{2}\left(\frac{1}{\kappa_{1}}+\frac{1}{\kappa_{2}}+\frac{L}{\lambda_{2}}\right)(t-L)^{2}-\frac{\lambda_{1}}{\kappa_{1}{ }^{2}} t+\left(\frac{\lambda_{1}}{\kappa_{1}{ }^{2}}\right. \\
& \left.+\frac{L}{\kappa_{1}}+\frac{L}{\kappa_{2}}\right)(t-L)-\frac{\lambda_{1}{ }^{2}}{\kappa_{1}{ }^{3}}\left\{e^{-\frac{\kappa_{1}}{\lambda_{1}} t}-e^{-\frac{\kappa_{1}}{\lambda_{1}}(t-L)}\right. \\
& \left.+u(t-L)-1\}-\frac{L \lambda_{1}}{\kappa_{1}{ }^{2}}\left\{u(t-L)-e^{-\frac{\kappa_{1}}{\lambda_{1}}(t-L)}\right\}\right]
\end{aligned}
$$

where $x(0)=0 \quad$ as $t=0$

$$
\begin{gathered}
x(L)=a_{L}^{\ulcorner} \frac{L^{3}}{6 \lambda_{2}}+\frac{1}{2}\left(\frac{1}{\kappa_{1}}+\frac{1}{\kappa_{2}}\right) L^{2}-\frac{\lambda_{1}}{\kappa_{1}{ }^{2}} L \\
\left.-\frac{\lambda_{1}{ }^{2}}{\kappa_{1}{ }^{3}}\left(e^{-\frac{\kappa_{1}}{\lambda_{1}} L}-1\right)\right] \text { as } t=L
\end{gathered}
$$

and $\quad x(\infty)=\infty \quad$ as $t=\infty$

These results show the same tendency that is shown by results of research in the field of rheology and by Watanabe's results $\left.\_2\right]$ of movement expression of viscous and elastic matter by intermediate differetial quantity.

3-1-2 Frequency characteristics

Frequency characteristic corresponds to the results of a test of a repeated load. It follows, then, that a four-element model has a mechanical inconsistency. Therefore, we approximate the transfer function of fibers to the Voigt's model. Then the transfer function of a test piece with extension input is obtainable as follows:

$$
G_{p}(s)=\kappa(T s+1)
$$

Substituting this equation into eq. (5), we get the transfer function $G_{V_{0 f}}(s)$ of the whole system as follows :

$$
G_{V o f}(s)=\frac{K_{c}}{a_{1} s^{2}+b_{1} s+c_{1}} e^{-L S}
$$

where $a_{1}=T_{M}-\kappa K_{D} L T$

$$
\begin{aligned}
& b_{1}=1-\kappa K_{D}(L-T) \\
& c_{1}=\kappa K_{D}
\end{aligned}
$$

Hence, the frequency response is

$$
\begin{gathered}
\left|G_{V o f}\right|=\frac{K_{c}}{\sqrt{\left(c_{1}-a_{1} \omega^{2}\right)^{2}+\left(\overline{b_{1} \omega}\right)^{2}}} \\
N \mathrm{~dB}=20 \log _{10} K_{c}-10 \log _{10}\left\{\left(c_{1}-a_{1} \omega^{2}\right)^{2}+\left(b_{1} \omega\right)^{2}\right\} \\
\phi=-\tan ^{-1} \frac{b_{1} \omega}{c_{1}-a_{1} \omega^{2}}-\angle L \omega
\end{gathered}
$$

where $\phi=0^{\circ}$ as $\omega=0$

$$
\text { and } \phi=-\infty^{\circ} \text { as } \omega=\infty
$$

The Bode diagram obtainable from these equations. 
is Fig. 10, where each gain constant is 1 , the time constant and the dead time of the servo-motor are $T_{M}=0.029 \mathrm{sec}$ and $L=0.014 \mathrm{sec}$, respectively, and a value obtained from a transience-response test is used as the time constant of a fiber. This example concerns Nylon, where $T_{M}=0.030 \mathrm{sec}$. However, allowing for temperature dependence, we use a value at $20^{\circ} \mathrm{C}$, the temperature usually used.

Murakami $\left.\_1\right]$ obtained the following fiber-model frequency response by using a load as input :

$$
\begin{array}{ll}
G \mid=\frac{1}{\kappa \sqrt{ } 1+(T \omega)^{2}} & \cdots \cdots \cdots(36) \\
N \mathrm{~dB}=-20 \log _{10} \kappa-10 \log _{10}\left\{1+(T \omega)^{2}\right\} & \cdots \cdots \cdots(37) \\
\phi=-\tan ^{-1} T \omega & \cdots \cdots \cdots(38\}
\end{array}
$$

where $\dot{\phi}=0^{\circ}$ as $\omega=0$

and $\dot{\phi}=-90^{\circ}$ as $\omega=\infty$

3-2. Analyzing Extension Input Test

The transfer function of our tester is separable from the transfer functioin of the whole system with a test piece inserted in it, because the transfer function $G_{p}(s)$ of a test piece is outside of the feed back loop in the block diagram of the tester (see Fig. 7). In other words, i) the transfer function of the whole system is

$$
G_{x}(s)=\begin{gathered}
K_{H} G_{p}(s) \\
T_{M} s^{2}+\left(1-K_{B} L\right) s \div K_{B}
\end{gathered} e^{L S}
$$

and ii) the transfer function of the tester alone is

$$
G_{A x}(s)=T_{M} s^{2}+\left(1-K_{B} L\right) s \cdots K_{B} e^{L s}
$$

Therefore, pick up the input signal from the output terminal of the feedback loop of the system and compare it with the final output of the system. We can then obtain the caracteristic of only a test piece freed from the influence of the tester. The dynamic characteristic of a fiber model in this case is as described in what follows.

3-2-1. Transience characteristic

Eq. (22) gives the transfer function of a test piece where extension input is used. Substitute the follow. ing :

$$
\kappa_{1} / \lambda_{1}=1 / T_{1}, \quad \kappa_{2} / \lambda_{2}=1 / T_{2}
$$

into that equation, then transform it so that it will be:

$$
\begin{aligned}
& G_{p}(s)=\frac{\kappa_{2}\left(s+\frac{1}{T_{1}}\right) s}{\left.s-\frac{1}{2}\left(\begin{array}{c}
1 \\
T_{1}+\frac{1}{T_{2}}+\frac{\kappa_{2}}{\lambda_{1}}
\end{array}\right)\right\}^{2}} \\
& +\left\{\sqrt[1]{T_{1} T_{2}}-\frac{1}{4}\left(T_{1}+\frac{1}{T_{2}-\kappa_{2}} \lambda_{1}\right)^{2}\right\}^{2}
\end{aligned}
$$

This new equation gives a different output response depending on whether the sign within the radical sign of the constant terms of the denominator is positive or negative. Let us, then, examine the

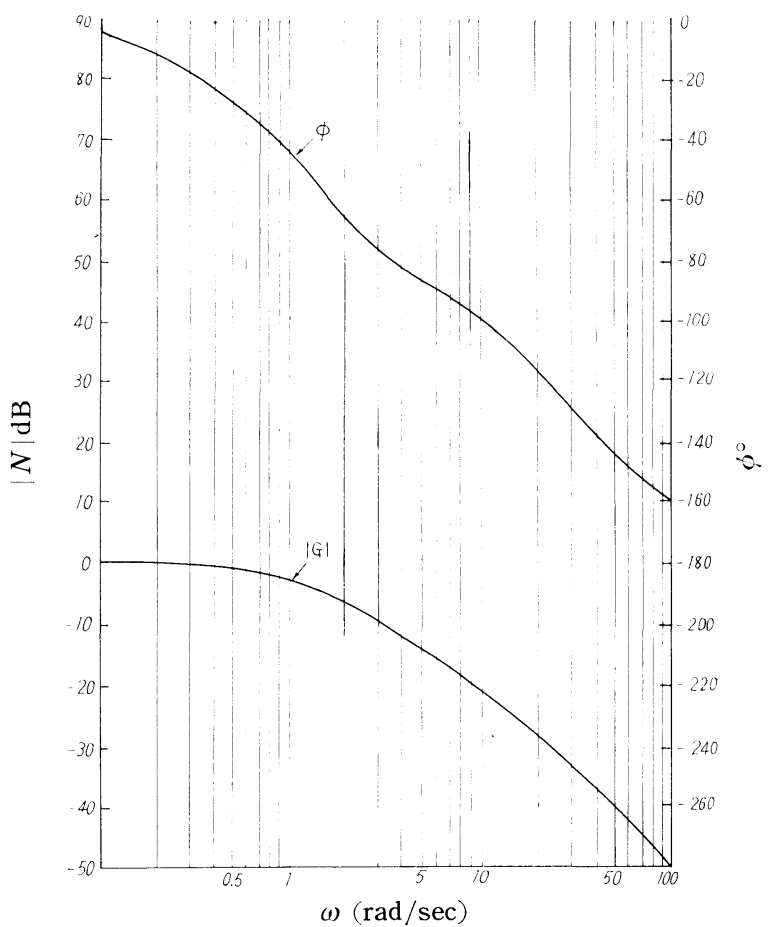

Fig. 10 Bode diagram (closed-loop) of fiber model calculated on load input test

sign within a radical sign. First, we compare $\begin{gathered}1 \\ T_{1} T_{2}\end{gathered}$ and ${ }_{4}^{1}\left(\begin{array}{cc}1 & 1 \\ T_{1} & T_{2}\end{array}\right)^{2}$, ignoring $\kappa_{2} / \lambda_{1}$ for the present. Apply. ing the principle that an arithmetical mean exceeds a geometrical mean, we get the following equation:

$$
\begin{gathered}
T_{1}{ }^{2}+T_{2}{ }^{2} \\
\quad 2
\end{gathered} T_{1} T_{2}
$$

This inequality can be transformed into:

$$
\frac{1}{4}\left(\begin{array}{c}
1 \\
\left.T_{1}+\frac{1}{T_{2}}\right)^{2} \geqq T_{1} T_{2}
\end{array}\right.
$$

$\kappa_{2} / \lambda_{1}$ in eq. (41) being a positive real number, the inside of the radical sign is always negative.

Therefore, the output response in this case takes the form of $\sinh x(t)$. Assuming $\kappa_{1}=\kappa_{2}=\kappa, \lambda_{1}=\lambda_{2}=\lambda$ and $\lambda / \kappa=T$ in eq. (22), we get the output response corresponding to various types of extension input :

(a) Where $x(t)=u(t)$

The output response corresponding to step-type extension input makes the stress relaxation curve shown in Fig. 11 (a) and is expressible by the following equation :

$$
f(t)=\kappa e^{-\frac{3}{2 T^{t}}}\left(\cosh \underset{2 T}{\sqrt{5}} t-\frac{1}{\sqrt{5}} \sinh \frac{\sqrt{5}}{2 T} t\right)
$$

where $f(0)=\kappa$

and $\quad f(\infty)=0$ 

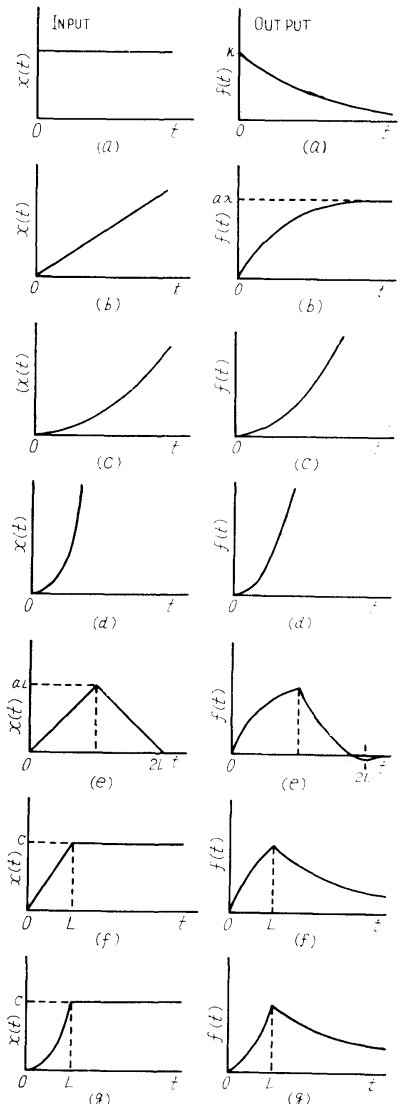

Fig. 11 Output load response curve for several input extensions

However, extension input of the step type is conceivable only theoretically and cannot be brought into being. Therefore, it is approximated, in practice, by combined extension input like (f), which will be dealt with later.

(b) Where $x(t)=a t$

The transience characteristic in this case is the same as in a test using the conventional tester of constant-speed extension type, as Fig. 11(b) shows, and its output response is expressible thus:

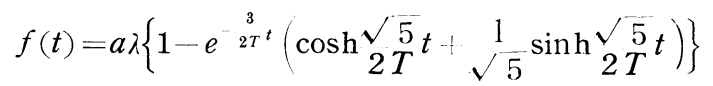

where $f(0)=0$ as $t=0$

and $f(\infty)=a \lambda$ as $t=\infty$

(c) Where $x(t)=\frac{1}{2} g t^{2}$

If we load constant-accelerative extension input by using the natural descent of a material (see Fig. 11 (c) ), we get the following output response curve :

$$
\begin{aligned}
f(t) & =g \lambda_{\llcorner} t-2 T \backslash 1-e^{-\frac{3}{2 T^{t}}}\left(\cosh \frac{\sqrt{5}}{2 T} t\right. \\
& \left.\left.+\frac{2}{\sqrt{5}} \sinh \frac{\sqrt{5} t}{2 T} t\right)\right\}
\end{aligned}
$$

where $f(0)=0$ and $f(\infty)=\infty$

(d) Where $x(t)=\frac{a}{6} t^{3}$

The output response curve corresponding to highspeed extension input is, as shown in Fig. 11 (d), as follows :

$$
\begin{aligned}
f(t) & =a_{\kappa} T\left[\frac{1}{2} t^{2}-2 T t+5 T^{2}-T^{2} e^{-3} T^{t}\left\{5 \left(\cosh \frac{\sqrt{5}}{2 T} t\right.\right.\right. \\
& \left.\left.\left.-3 \frac{3}{\sqrt{5}} \sinh \frac{\sqrt{5}}{2 T} t\right)+\frac{26}{\sqrt{5}} \sinh \frac{\sqrt{5}}{2 T} t\right\}\right] \cdots \cdots \cdots(47)
\end{aligned}
$$

where $f(0)=0$ and $f(\infty)=\infty$

(e) Where

$$
x(t)=a\{t-2(t-L) u(t-L)+(t-2 L) u(t-2 L)\}
$$

The output response curve corresponding to extension input of the triangular-wave type shown in Fig. 11 (e) is :

$$
\begin{aligned}
& f(t)=a \lambda_{L}^{\ulcorner} 1-2 u(t-L)+u(t-2 L)-e^{2} \tau^{t}\left(\cosh \frac{\sqrt{5}}{2} t\right. \\
& \left.+\frac{1}{\sqrt{5}} \sinh \frac{\sqrt{5}}{2 T} t\right)+2 e^{{ }^{2} T}+{ }^{L}\left\{\cosh \frac{\sqrt{5}}{2 T}(\mathrm{t}-L)\right. \\
& \left.+\frac{1}{\sqrt{5}} \sinh \frac{\sqrt{5}}{2}(t-L)\right\}-e^{2^{3} T^{2}{ }^{2 L}}\left\{\cosh \frac{\sqrt{5}}{2 T}(t\right. \\
& \left.\left.-2 L)+\frac{1}{\sqrt{5}} \sinh \frac{\sqrt{5}}{2}(t-2 L)\right\}\right] \quad \cdots \cdots \cdots \cdot 48
\end{aligned}
$$

where $f(0)=0$

$$
\begin{aligned}
& f(L)=a \lambda\left\{1-e^{2 T^{2}}\left(\cosh _{2}^{\sqrt{5}} L+\frac{1}{\sqrt{5}} \sinh \underset{2 T}{\sqrt{5}} L\right)\right\} \\
& f(2 L)=-a \lambda_{L}^{r} e^{T^{3} L}\left(\cosh \frac{\sqrt{5}}{T} L+\frac{1}{\sqrt{5}} \sinh { }_{T}^{\sqrt{5}} L\right) \\
& \left.-2 e^{{ }^{3} T^{L}}\{\cosh \underset{2 T}{\sqrt{5}} L+\sqrt{\sqrt{5}} \sinh \underset{2 T}{\sqrt{5}} L\}+1\right]
\end{aligned}
$$

and $f(\infty)=0$

The reason why the output response at $t=2 L$ enters the negative side in this figure is that the output response is expressed in the form of $\sinh x(t)$, as stated at the begeinning of this section.

(f) Where $\quad x(t)=a t\{1-u(t-L)\}+c u(t-L)$

The output response corresponding to the combined input shown Fig. 11 (f) is given below. This is an example showing that extension input of the step type is unobtainable in practice and is actually extension input of the type shown below:

$$
\begin{aligned}
& f(t)=a \lambda\left[1-u(t-L)-e^{2 T^{t}}\left(\cosh \frac{\sqrt{5}}{2 T} t\right.\right. \\
& \left.+\sqrt{5} \sinh 2 \frac{\sqrt{5}}{2 T} t\right)+e^{-\frac{3}{2 T}(t-L)}\left(\cosh \frac{\sqrt{5}}{2 T}(t-L)\right.
\end{aligned}
$$




$$
\left.\left.+\frac{1}{\sqrt{5}} \sinh \frac{\sqrt{5}}{2 T}(t-L)\right\}\right]
$$

where $\quad f(0)=0$

$$
f(L)=a \lambda\left\{1-e^{-3}{ }_{2 T}^{L}\left(\cosh _{2 T}^{\sqrt{5}} L+\sqrt{5} \sinh \frac{\sqrt{5}}{2 T} L\right)\right\}
$$

and $f(\infty)=0$

(g) Where $x(t)=\frac{1}{2} g t^{2}\{1-u(t-L)\}+c u(t-L)$

The output response corresponding to the combined extension input shown in Fig. $11(\mathrm{~g})$ is :

$$
\begin{aligned}
& f(t)=g \lambda(\{1-u(t-L)\}(L-2 T) \\
& +2 T_{L}^{-e^{-3 T^{t}}}\left(\cosh \frac{\sqrt{5}}{2 T} t+\underset{\sqrt{5}}{2} \sinh \frac{\sqrt{5}}{2 T} t\right) \\
& \left.-e^{2 T}{ }^{3}+L:\left\{\cosh _{2} \frac{\sqrt{5}}{T}(t-L)+\frac{2}{\sqrt{5}} \sinh (t-L)\right\}\right] \\
& +L e^{3}{ }_{2 T}^{3}+L \cdot\left\{\cosh _{2 T}^{\sqrt{5}}(t-L)+\frac{1}{\sqrt{5}} \sinh (t-L)\right)
\end{aligned}
$$

where $f(0)=0$

$$
\begin{aligned}
f(L) & =g \lambda\left[L+2 T\left\{e ^ { - 3 } L \left(\cosh \frac{\sqrt{5}}{2 T} L\right.\right.\right. \\
& \left.\left.+\sqrt{5} \sinh \frac{\sqrt{5}}{2} L\right)-1\right]
\end{aligned}
$$

and $\quad f(\infty)=0$

\section{3-2-2. Frequency characteristic}

The characteristic of a test piece being separable if extension input is used, we approximate a fiber model to Voigt's model, as we did to obtain the frequency response of load input. The frequency response of the fiber model thus approximated is:

$$
\begin{array}{ll}
G=\kappa \sqrt{ } 1+(T \omega)^{2} & \cdots \cdots \cdots(51) \\
N \mathrm{~dB}=20 \log _{10} \kappa+10 \log _{10}\left\{1+(T \omega)^{2}\right\} & \cdots \cdots \cdots(52) \\
\phi=\tan ^{-1} T() & \cdots \cdots \cdots(53)
\end{array}
$$

where $\phi=0^{\circ} \quad$ as $\omega=0$

and $\quad \dot{\phi}=90^{\circ}$ as $(1)=\infty$

Note that the phase angle of the frequency response corresponding to extension input, unlike the phase angle of the frequency response corresponding to load input, leads. This has been experimentally proved, as will be shown later.

Fig. 12 is a Bode diagram obtained by calculation concerning a fiber model, where each gain constant is taken as 1 and the time constant and dead time of the servo-motor are as given earlier. The time constant of a fiber is a value obtained from the follow. ing transience response tests made at $20^{\circ} \mathrm{C}$ :

Nylon : $0.030 \mathrm{sec}$

Tetoron : $0.055 \mathrm{sec}$

Raw silk : $0.012 \mathrm{sec}$

\section{Results of Experiments Discussed}

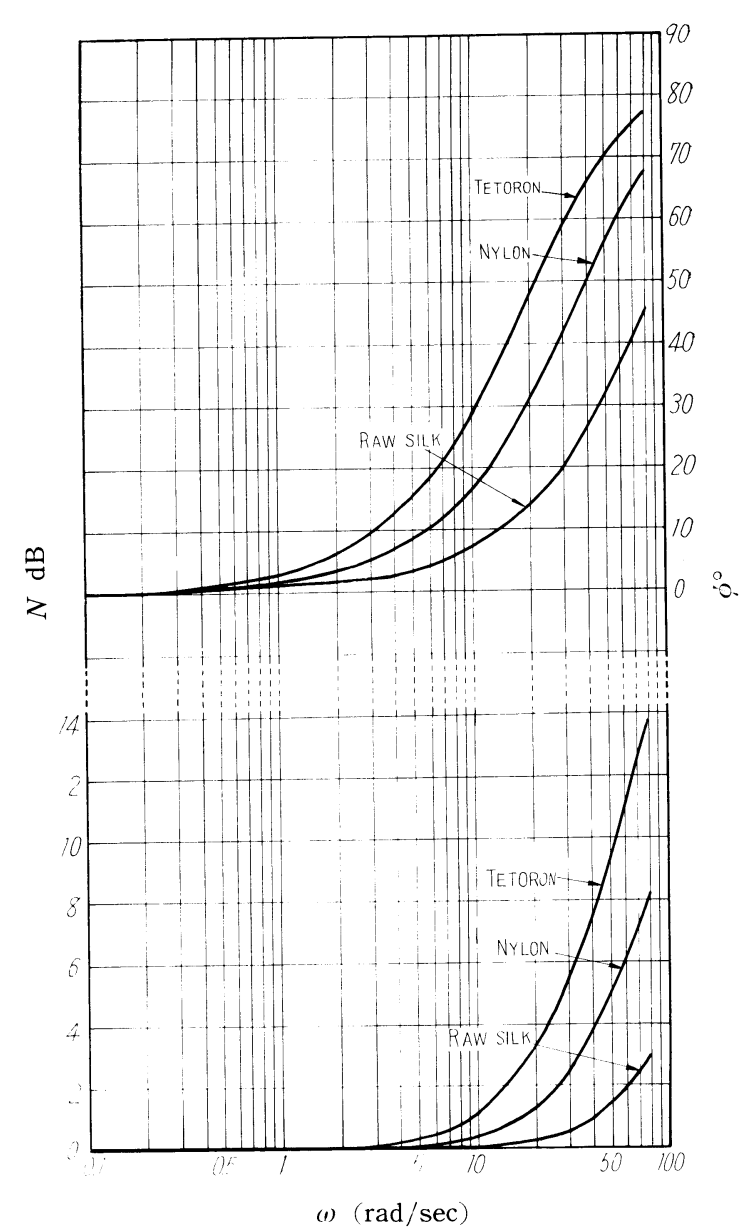

Fig. 12 Bode diagram of feed back system for fiber model calculated on extension input test

4-1. Measuring with Our Tester under Load Input

Our tester can be used for tests in which loads of arbitrary functions are imposed on test pieces, but the present article concerns the results of measurement of output extension responses corresponding to input of various types: step, constant-speed, constant-acceleration, high-speed and repeated-load.

(Test piece)

1) Rubber

(Fineness)

2) Spandex

3) Nylon $6470 \mathrm{~d}$ $226.5 \mathrm{~d} \times 8$

4) Tetoron $67 \mathrm{~d}$

5) Raw silk $75.7 \mathrm{~d}$

$22.7 \mathrm{~d}$

Conditions of tests : $20^{\circ} \mathrm{C}, 65 \% \mathrm{RH}$

Length of test pieces (except Spandex and Rubber $60 \mathrm{~cm}$

Length of Spandex and Rubber: $10 \mathrm{~cm}$

Figs. 13-17 show the relation between the input 

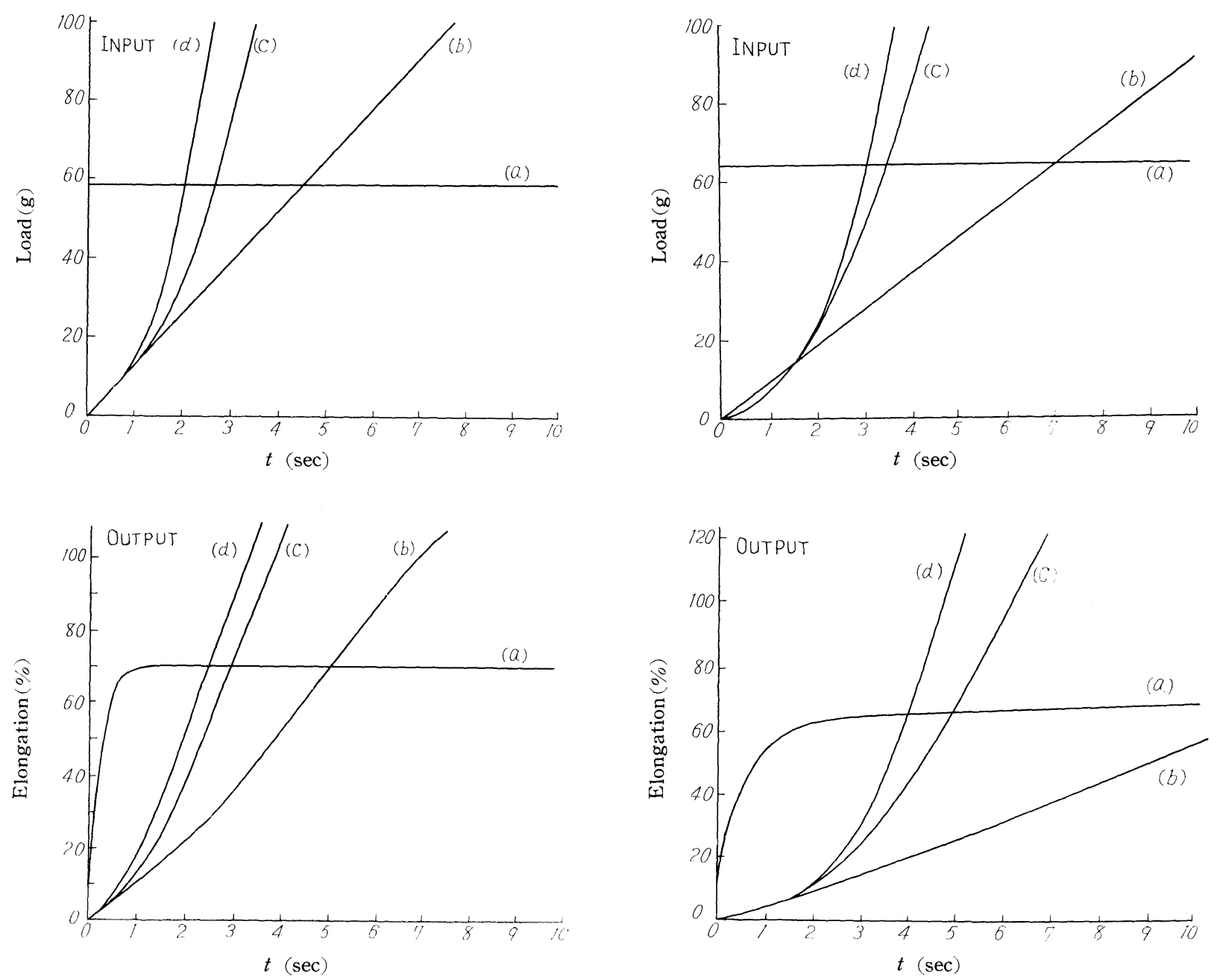

Fig. 13 Load- $t$ curve of rubber tested under load input

Fig. 14 Load-t curve of Spandex tested under load input

load and output displacement (extension) of each test piece. (a), (b), (c) and (d) in the figure show the functional forms of input corresponding to the various types of input.

The different kinds of test materials exhibit their respective characteristics in these figures, but as a general rule, they show the same tendency as the theoretical curve given in (a) - (d) in 3-1-1.

Load-extension curves are directly obtainable by using the $\mathrm{X}-\mathrm{Y}$ recorder, but we measured and, show separately, input load and output extension to clarify the relation to the theoretical curve just mentioned.

Figs. 18-22 are stress-strain curves obtained from experimental results. They differ according to the type of input signals, as clearly shown in these figures. We leave inquiry into these differences to experts.

Now to give the results of experiments which were tantamount to tests of repeated stress (repeated-stress tests have been rare). Remember that test pieces tested under a certain amount of stress, plus repeated, fluctuating stress, creep and change gradually in length. A mechanism is needed that will roll up test pieces in by the lengths of their successive creeps so as to keep test pieces constant in length. This, however, is a subject for future inquiry.

Fig. 23 shows a phase curve obtained from the results of a frequency response test made under a repeated load of $0.02-5 \mathrm{c} / \mathrm{sec}$ obtained from sin waves generated by the analog computer. The dotted line is the phase curve obtained by putting a spring into the tester. The fact that the phase curve of every tested fiber has a smaller phase-lag than does the spring is explained as follows :

Take all gain constants as 1 to simplify eqs. (6) 

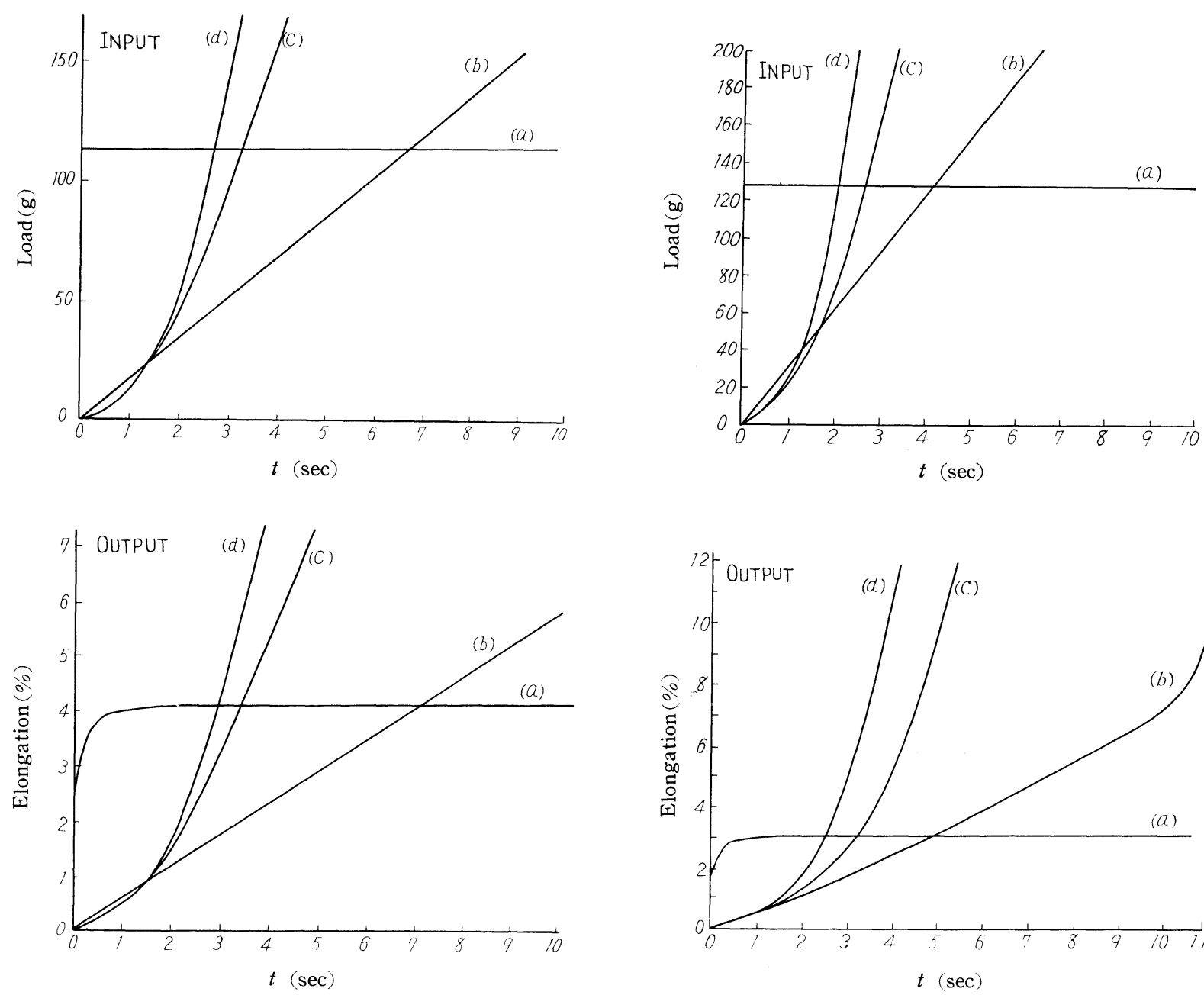

Fig. 15 Load-t curve of Nylon tested under load input

and (32) on the transfer function where a spring and a fiber (Voigt) model, respectively, are put into the tester under load input. The transfer function where a spring is inserted into the tester is, then :

$$
G_{s f}(s)=\frac{1}{T_{M j^{2}}+\left(1-K_{s} L\right) s+K_{s}} e^{-L s} \quad \cdots \cdots \cdots \cdot 54
$$

The transfer function where a fiber model is put into the tester under load input tester is :

$$
G_{V o f}(s)=\frac{1}{\left(T_{M}-\lambda L\right)} \frac{1}{s^{2}+(1+\lambda-\kappa L) s+\kappa^{e-L S} \cdots(55)}
$$

Damping coefficients $\zeta_{s}$ and $\zeta_{Y}$ obtained from these two equations when a spring is inserted into the tester under load input:

$$
\zeta_{s}=\frac{1-K_{s} L}{2 \sqrt{ } K_{s} T_{M}}
$$

and when a fiber model is put into the tester:

$$
\zeta_{Y}=\frac{1+\lambda-\kappa L}{2 \sqrt{\kappa}\left(T_{M}-\lambda L\right)}
$$

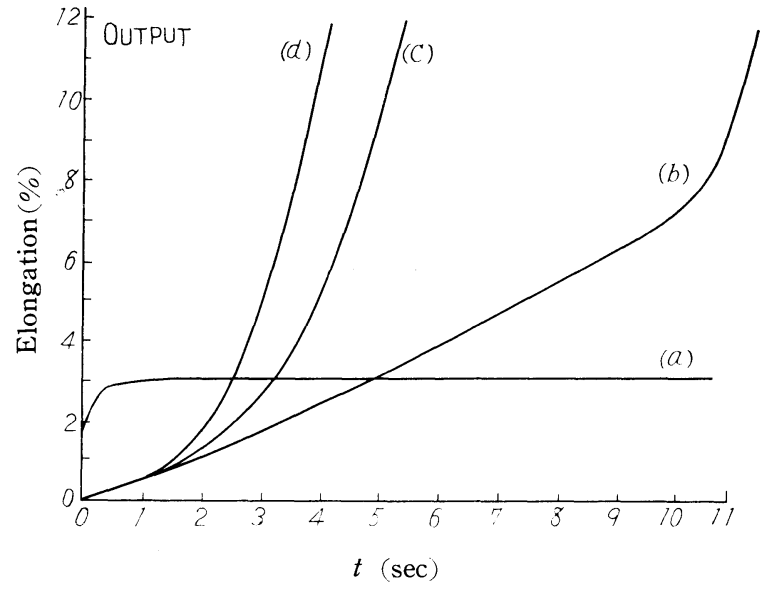

Fig. 16 Load-t curve of Tetoron tested under load input

Omitting the term of $e^{-L S}$ to simplify the equation leads to :

$$
\begin{aligned}
& \zeta_{s} \fallingdotseq \frac{1}{2} \quad \frac{1}{K_{s} T_{M}} \\
& \zeta_{Y} \doteqdot \begin{array}{c}
1+\lambda \\
2 \sqrt{ } \kappa T_{M}
\end{array}
\end{aligned}
$$

Now to look into the relation between phase-lag and damping coefficient. Eqs. (54) and (55) being both second-order transfer functions, it is theoretically clear that the larger the damping coefficient, the larger the phase-lag. Compare $\zeta_{s}$ and $\zeta_{Y}$ in eqs. (58) and (59) in size, assumming $K_{s}=\kappa$. Then :

$$
\zeta_{Y}>\zeta_{s}
$$

Conceivably, then, a fiber model has a larger phase-lag than a spring does. If we think of a spring as perfectly elastic matter within the limits of elasticity, a fiber model should have a larger phase-lag than does a spring, because a fiber model is subject 

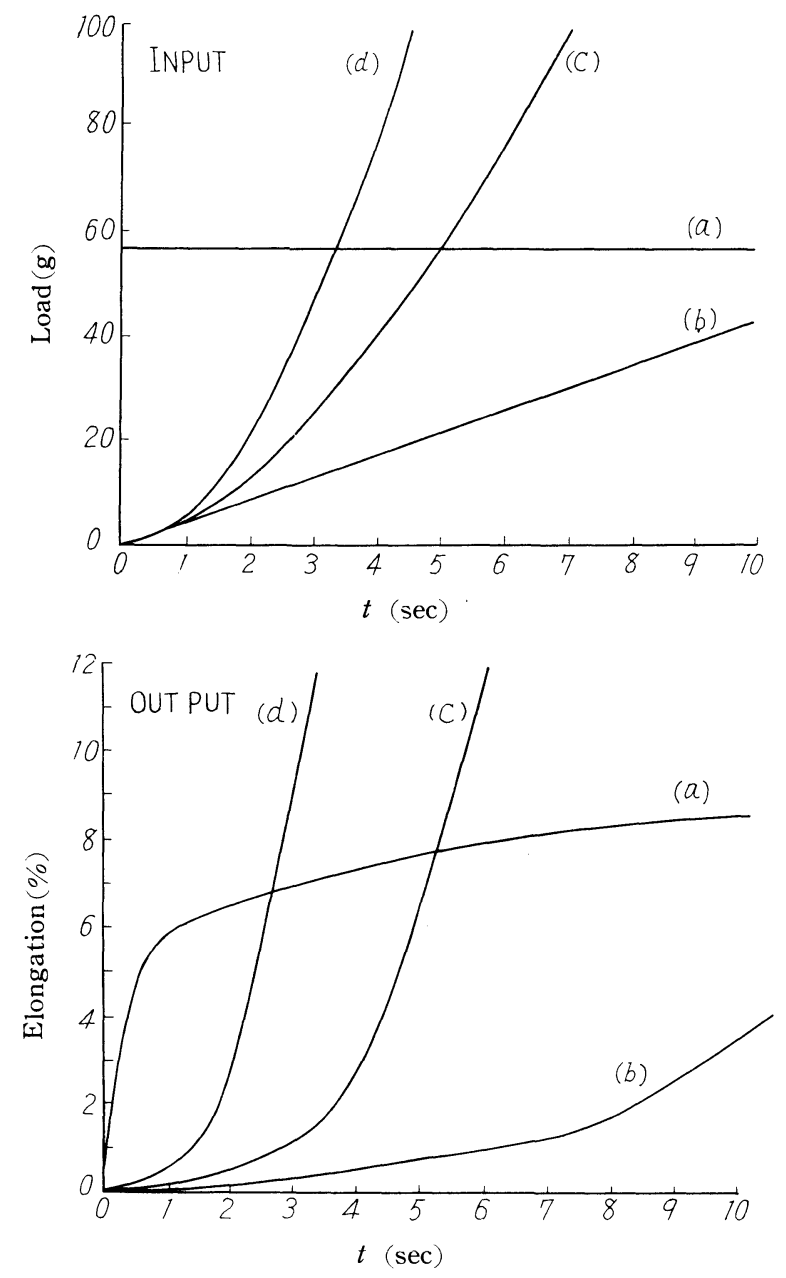

Fig. 17 Load- $t$ curve of Raw silk tested under load input

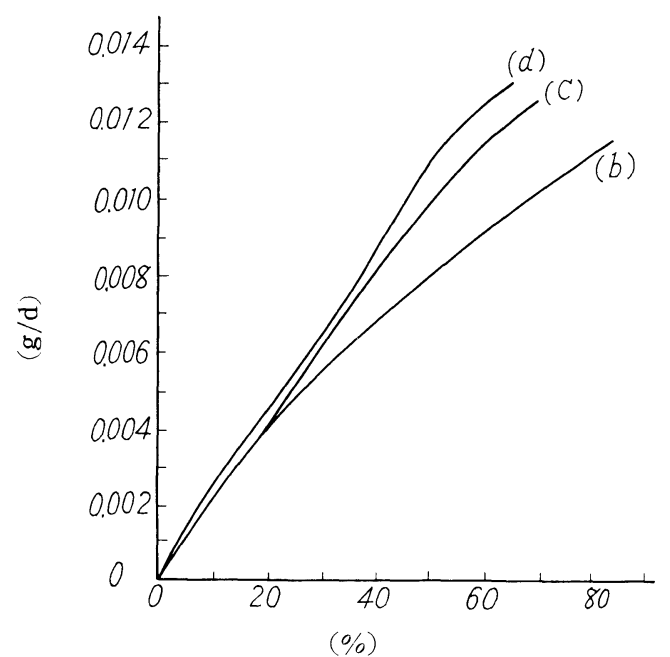

Fig. 18 Stress strain curve of rubber tested under load input

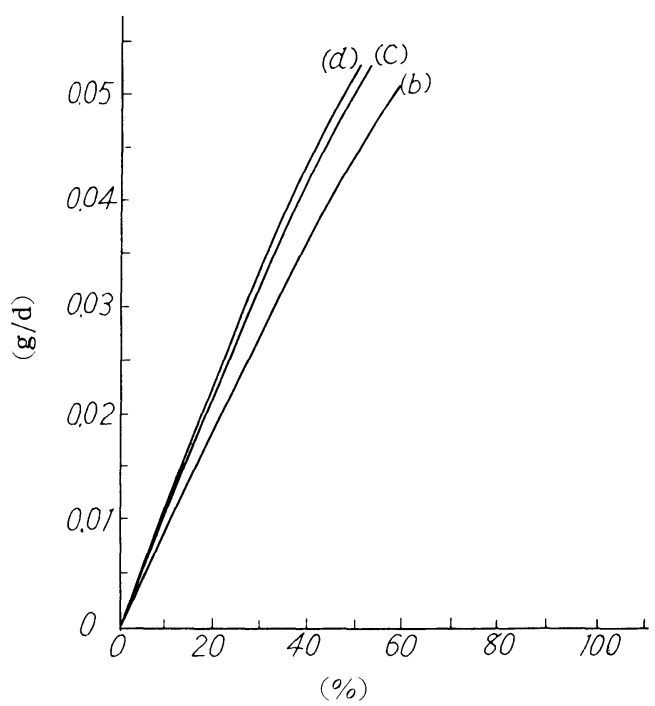

Fig. 19 Stress strain curve of Spandex tested under load input

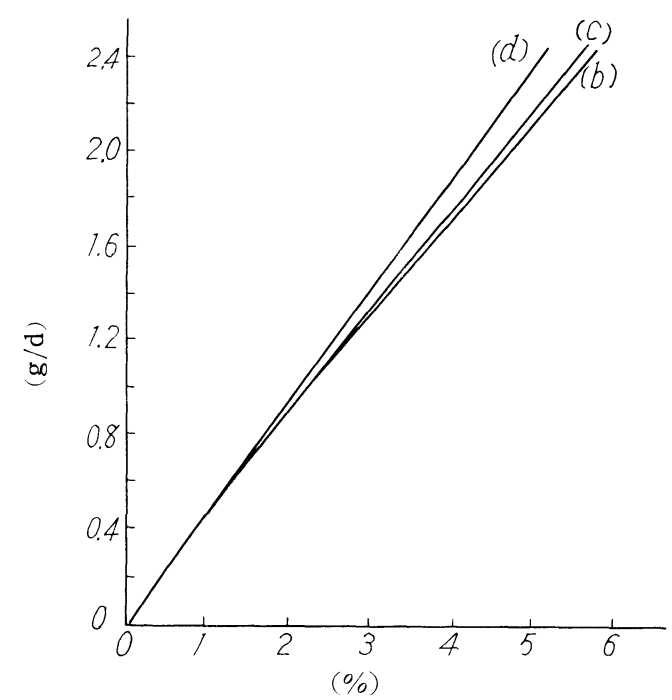

Fig. 20 Stress strain curve of Nylon tested under load input

to the influence of damper coefficient $\lambda$. Yet the experimental result given Fig. 23 shows the reverse tendency.

Therefore, revoking the previous assumption that $\kappa_{s}=\kappa$ and then assuming $K_{s} \neq \kappa$ and that the value of $\lambda$ in eq. (59) is small, we require that $K_{s}<\kappa$ as the prerequisite to $\zeta_{s}>\zeta_{\mathrm{r}}$. Fig. 23 shows that the following inequalities are required for, respectively, Tetoron, Raw silk, Nylon, etc. :

$$
\begin{aligned}
& \left.K_{s}<\kappa(\text { Nylon })<\kappa(\text { Raw silk or Tetoron }) \cdots \cdots \cdots 60\right) \\
& K_{s} \geqq \kappa(\text { for Spandex }) \\
& \cdots \cdots \cdots \cdots 61)
\end{aligned}
$$




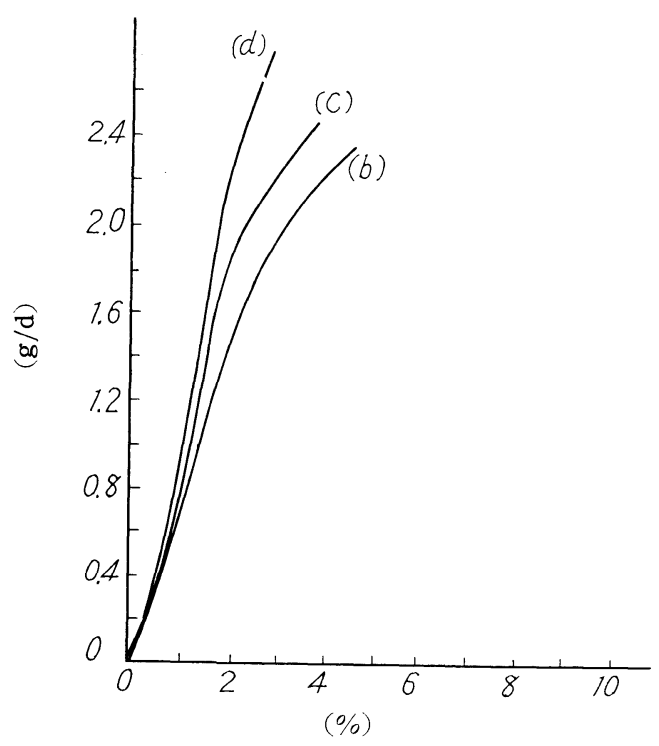

Fig. 21 Stress strain curve of Tetoron tested under load input

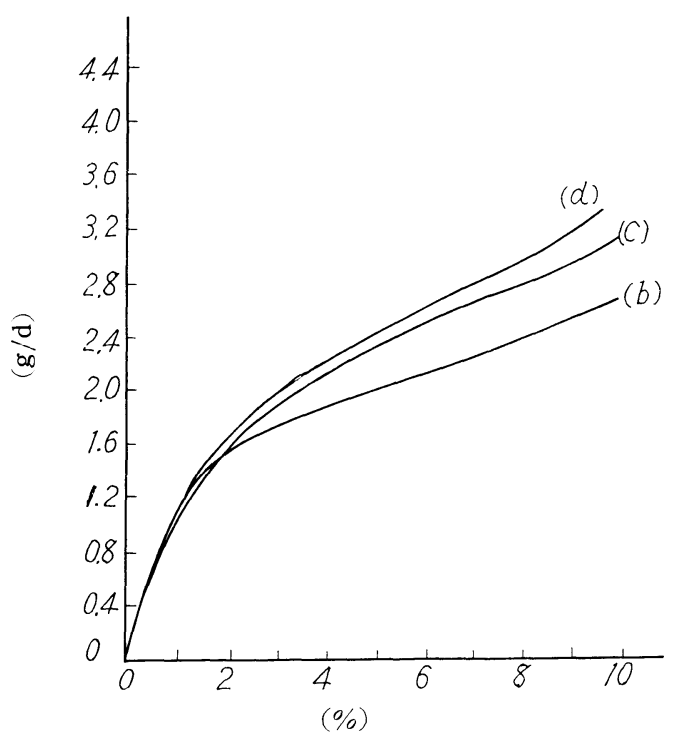

Fig. 22 Stress strain curve of Raw silk tested under load input

Investigation of these point involves measurement of the elastic constant of a fiber, which is a difficult task because a fiber is viscous. Nevertheless, we measured values close to the elastic constants of test pieces under a light load, with the following results :
Tetoron
$62.5 \mathrm{~g} / \mathrm{mm}$
Nylon
$22.7 \mathrm{~g} / \mathrm{mm}$
Spring
$5.9 \mathrm{~g} / \mathrm{mm}$
Spandex
$0.47 \mathrm{~g} / \mathrm{mm}$

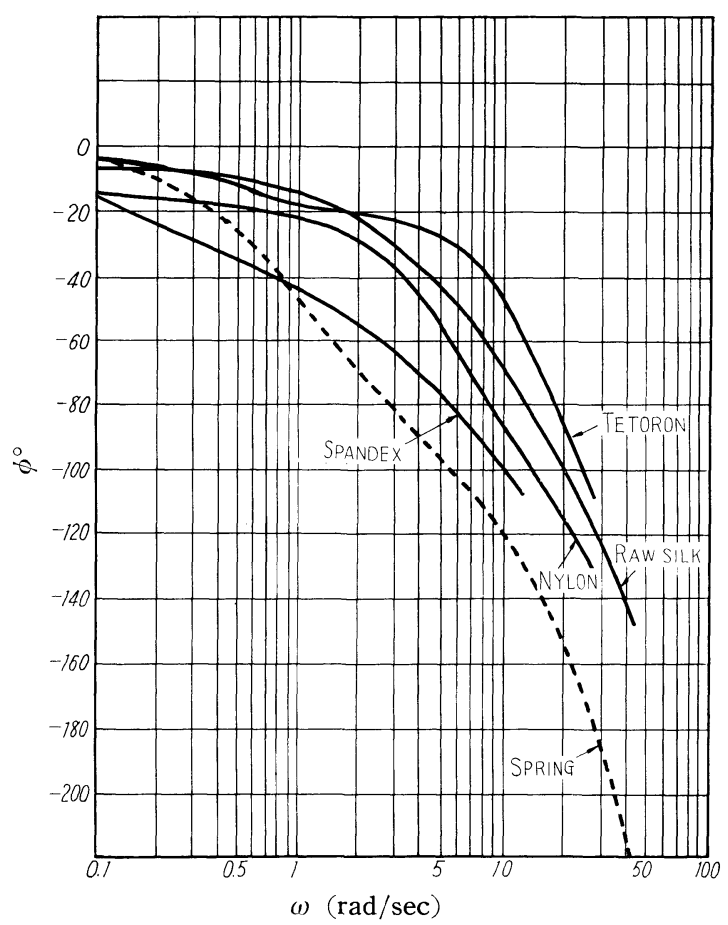

Fig. 23 Bode diagram (phase angle) of fiber tested under Load input

These values agree well with the assumptions in eqs. (60) and (61). Considering the phase curve of Fig. 23 in the light of this fact, we believe that the value of elastic constant $\kappa$ in Raw silk, Tetoron and Nylon has greater influence on the damping coefficient $\zeta_{Y}$ than does damper coefficient $\lambda$ in eq. (59); and that, when $K_{s}\left\langle\kappa, \zeta_{s}\right\rangle \zeta_{\mathrm{Y}}$. Presumably, therefore, these fibers are smaller in phase-lag than a spring. How. ever, Spandex does not differ much from a spring in phase-lag, notwithstanding $\kappa \leqslant K_{s}$.

This presumably explains why damper coefficient $\lambda$ of Spandex is larger in value than the similar coef. ficient of the other fibers. The explanation seems to be borne out by the fact, shown by Fig. 14 (a) (creep test results), that Spandex creeps measurably.

\section{4-2. Measurement by Extension Input Tester}

Instron and other conventional tester are good for, say, constant-speed extension tests. Our tester is good also for tests of input of other arbitrary functions. The present article gives the results of measurement of output responses corresponding to step, constantspeed, constant-acceleration, high-speed extension input and repeated extension inputs of fibers and rubber. The test materials and the condition for measurement are the same as those for the load input test reported upon earlier. 

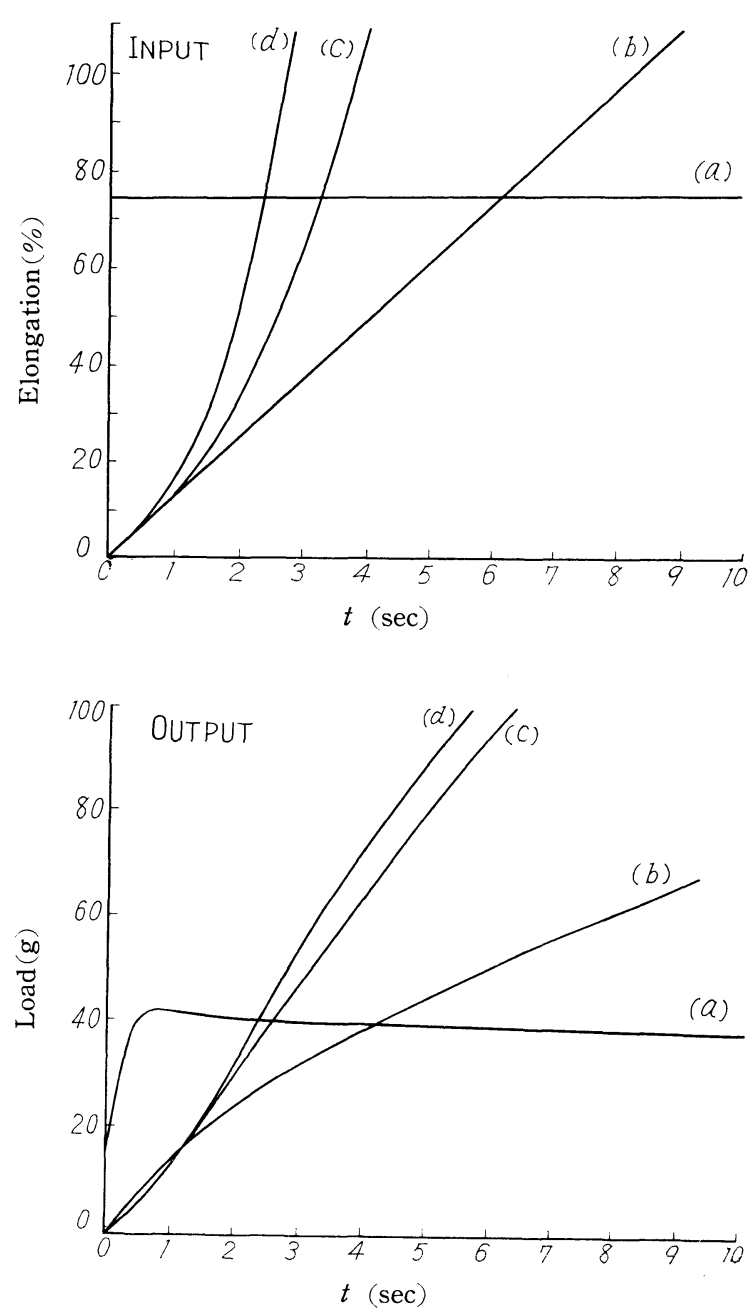

Fig. 21 Load $t$ curve of rubber tested under extension input

Figs. 24-28 show an output response curve corresponding to the input extension of each test material. (a), (b), (c) and (d) in the figures indicate the func. tion forms of various types of input: step, constantspeed, contant-acceleration and high-speed extension inputs. Strictly speaking, step input is unobtainable under extension input. Therefore, we use input combined in the same way as in (f) in 3-2-1 as an approximation of step input. The characteristics of the various fibers appear in the figures. The theoretical curves in the figure are simple ones based on VoigtMaxwell's four-element model. They do not agree with experimental curves and may, therefore, conceivably furnish a clue to investigating structural models of the fibers tested.

Figs. 29-32 are stress-strain curves obtained from experimental results. They seem to differ according to the type of input. The phase angle of a frequency
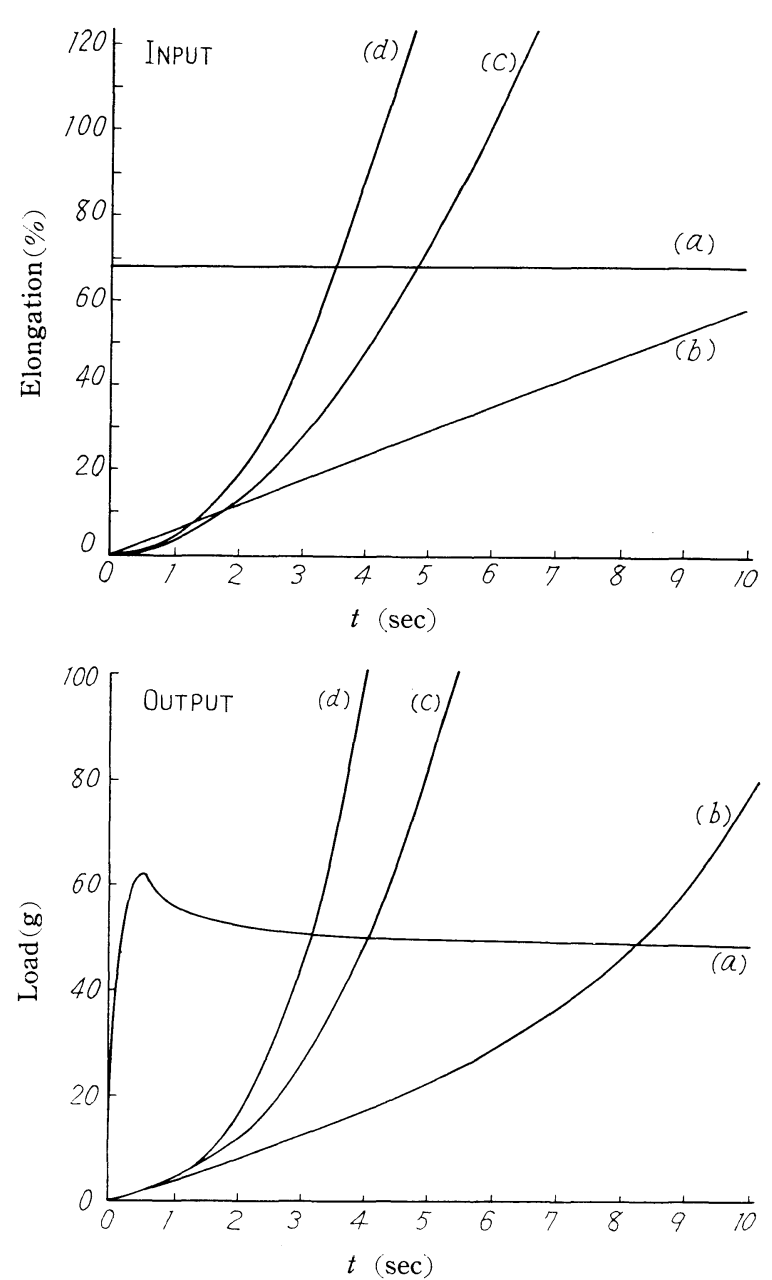

Fig. 25 Load $-t$ curve of Spandex tested under extension input

response in the extension input test to a fiber theoretically leads, as we said earlier. To investigate this theoretical point experimentally, we made frequency response tests of Nylon, Tetoron and Raw silk, with the results given in Fig. 34 .

Lest the results of the tests be affected by the dead time of the tester or by the lag of response, the input signal was taken from the output terminal of the feedback loop and made to correspond to the output signal. The phase angle shows lead phenomenon (a shift to positive) clearly in this figure, and exhibited a tendency similar to the calculated curve in Fig. 12.

\section{Examples of Applied Tests}

This article has been devoted, so far, to reporting 

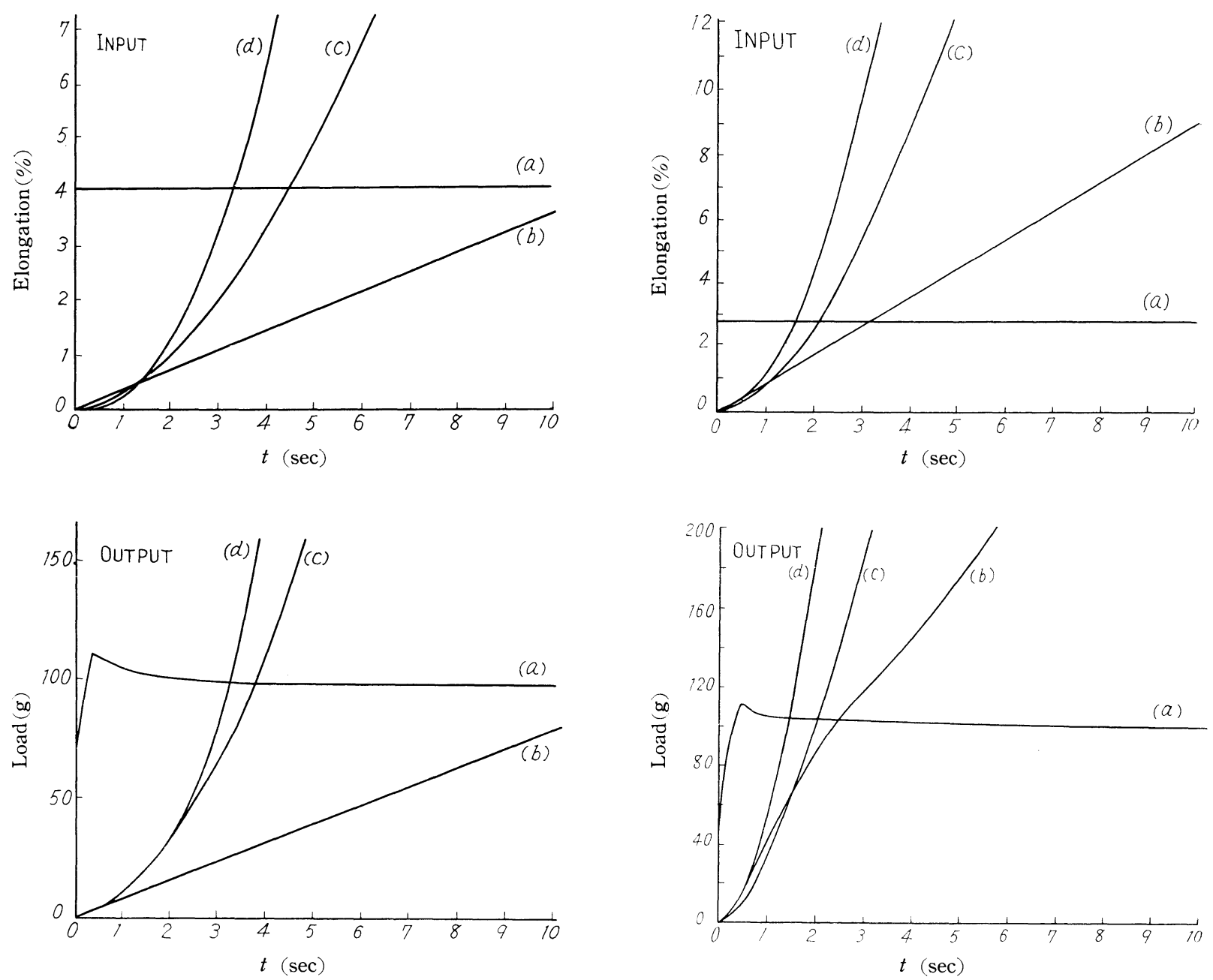

Fig. 26 Load $-t$ curve of Nylon tested under extension input

on tests with load or extension input given to the test pieces. The final aim of our all-purpose tester is to make tests under the so-called stress input. Some examples of applied tests, aside from their practical value, are given.

5-1 Method of Testing with Sectional Area of Test Piece Taken as Input.

Aiming at a test under stress input, we first dis. cuss a method of testing with the sectional area of the test piece changed according to the program. Let us show how to obtain the relation between extension and stress when we reduce the sectional area of the test piece at a constant speed in a tension test of rubber, plastics and the other materials. Fig. 35 is a block diagram of the tester used, where:

$G_{H X}(s)$ : transfer function of material by which force coming of the test piece is converted

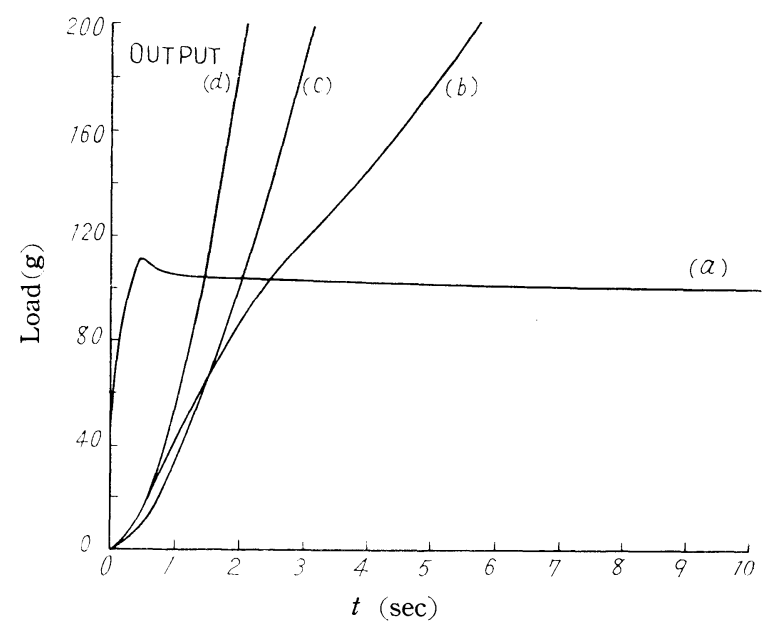

Fig. 27 Load $t$ curve of Tetoron tested under extension input

into horizontal component strain.

$G_{V F}(s)$ : transfer function of material by which force coming of the test piece is converted into a vertical component load (the force in the direction of the axis)

$K_{\mathrm{s}}$ : gain constant of the differential transformer $P$ : gain constant of potentiometer in an analog computer

The other symbols are the same as those in 2-21. The test pieces used differ somewhat in transfer function $G_{p}(s)$ from those referred to in the previous sections.

To use the area as input, we use an analog computer and integrate the voltage, which is proportional to horizontal strain $2 r_{1}$, by an integrator for conversion into input voltage $e_{i}{ }^{2}$ which takes the form of an area proportional to $r_{1}{ }^{2}$. Transmitting this input vol- 

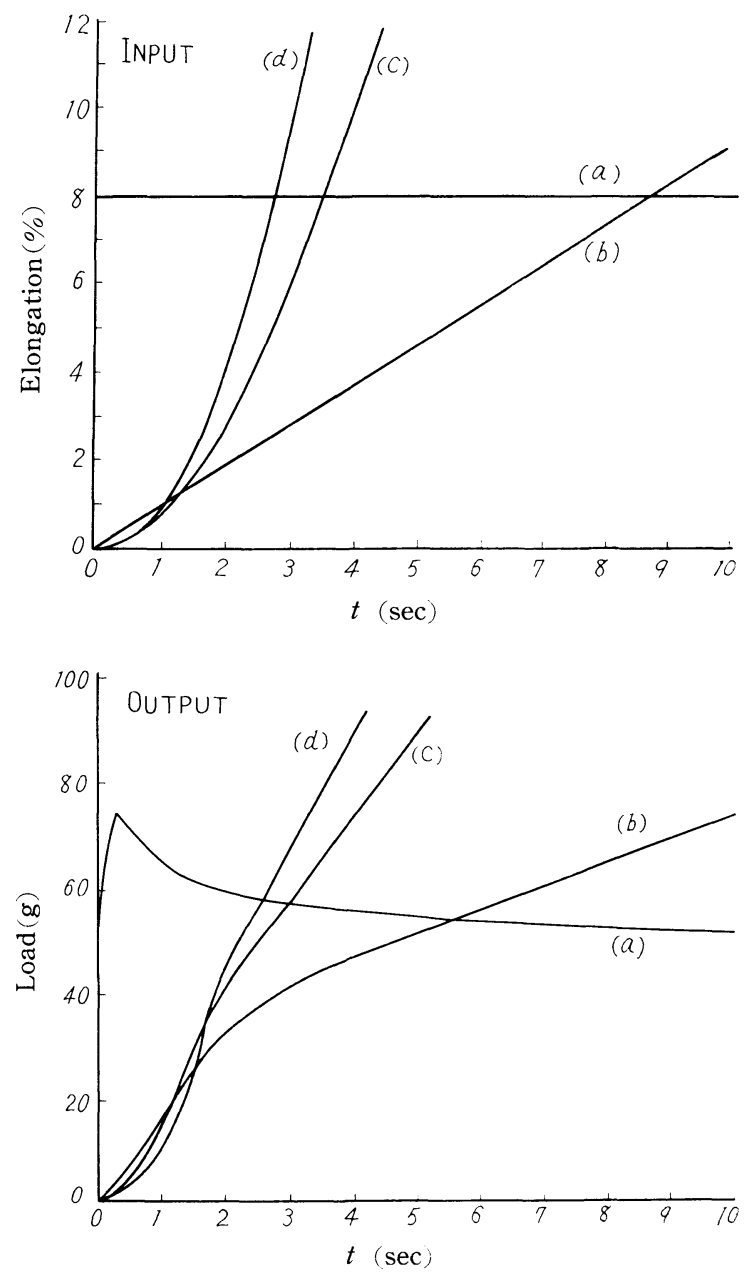

Fig. 28 Load $t$ curve of Raw silk tested under extension input

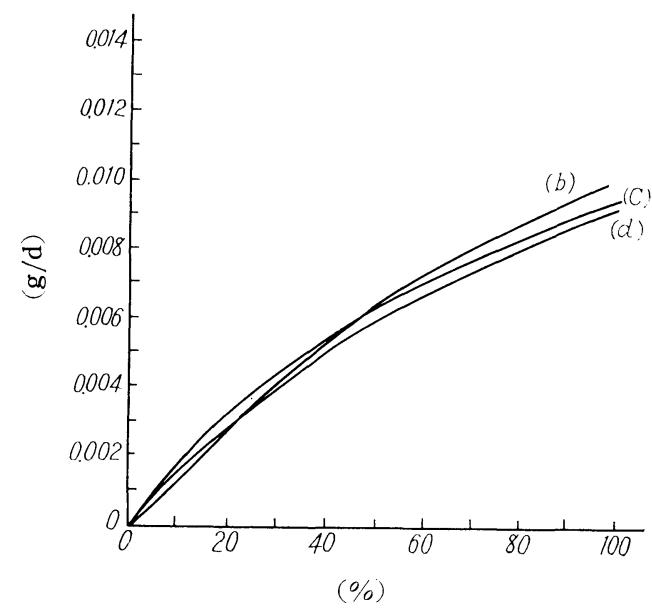

Fig. 29 Stress strain curve of rubber tested under extension input

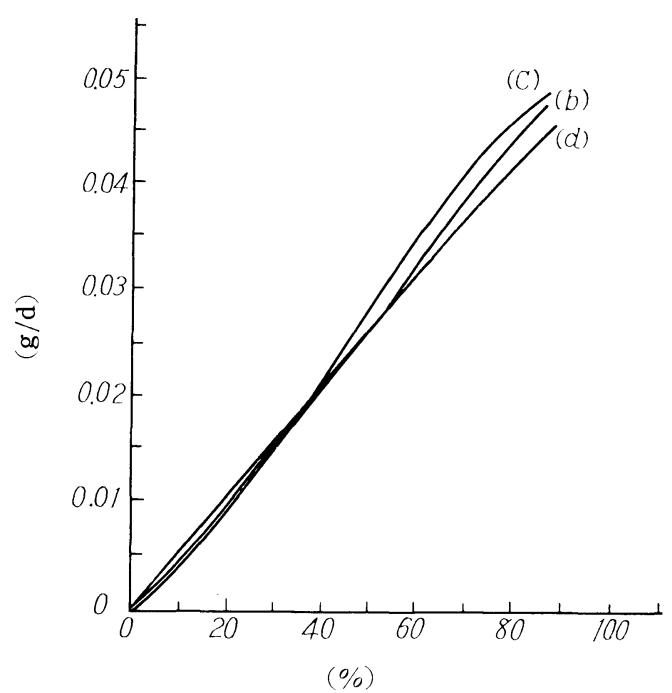

Fig. 30 Stress strain curve of Spandex tested under extension input

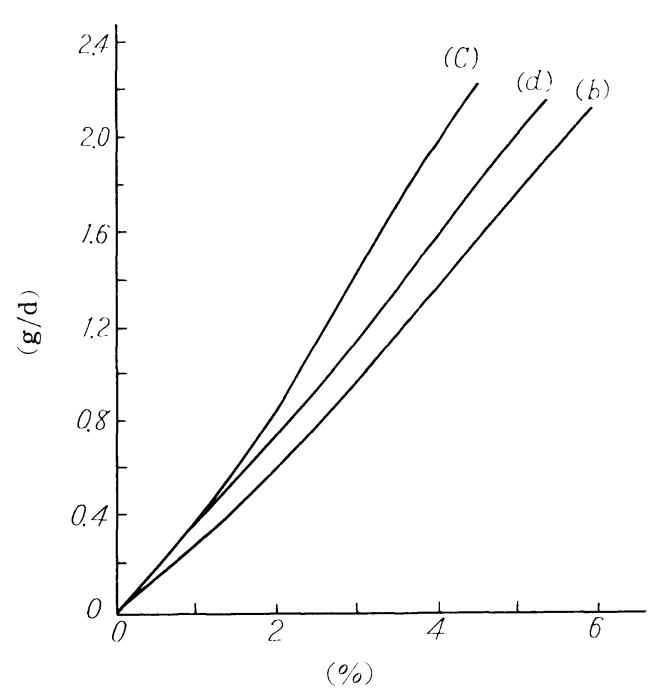

Fig. 31 Stress strain curve of Nylon tested under extension input

tage into the servo-motor through the servo-amplifier and driving the terminal of extension of the test piece, we can extend the test piece and transform its sectional area.

The horizontal component strain of the test piece is detected by the method shown in Fig. 26. Using the detecting pick-up, one side of which is the iron core of the differential transformer, we bring it into contact with the middle of the test piece. Here the head of the detecting pickup is given measurement pressure by a mild spring and is allowed to follow the 


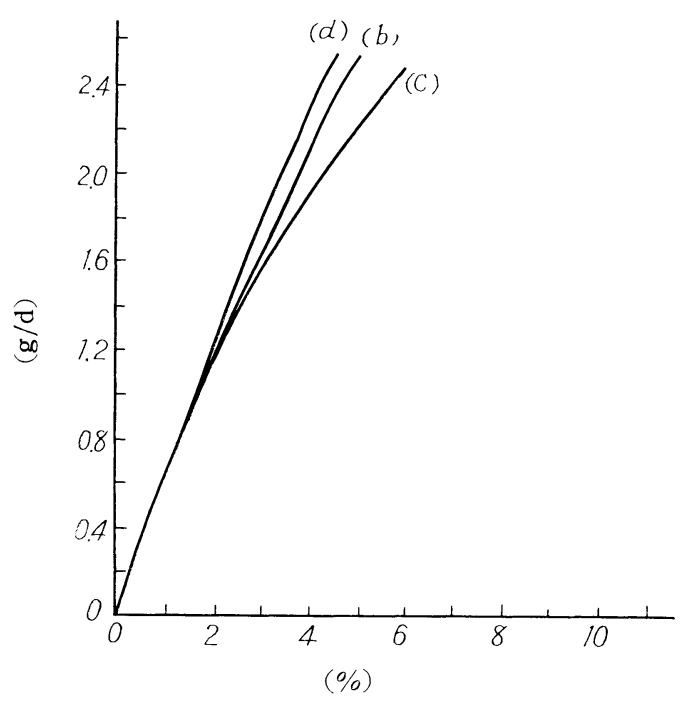

Fig. 32 Stress strain curve of Tetoron tested under extension input

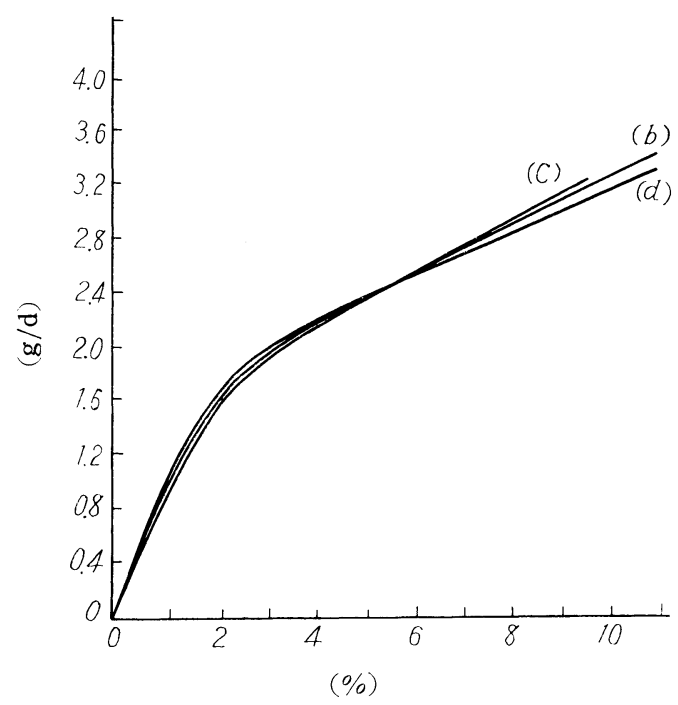

Fig. 33 Stress strain curve of Raw silk tested under extension input

horizontal component strain of the test piece.

The contact point of the detecting pickup shifts with the extension of the test piece This is rectified by position control by the method explained in 5-2. The voltage of the horizontal component strain detected by the differential transformer is converted, through an integrator, into a voltage proportional to the area and compared with the input voltage mentioned earlier.

Potentiometer $\mathrm{P}$ must be adjusted in gain before-

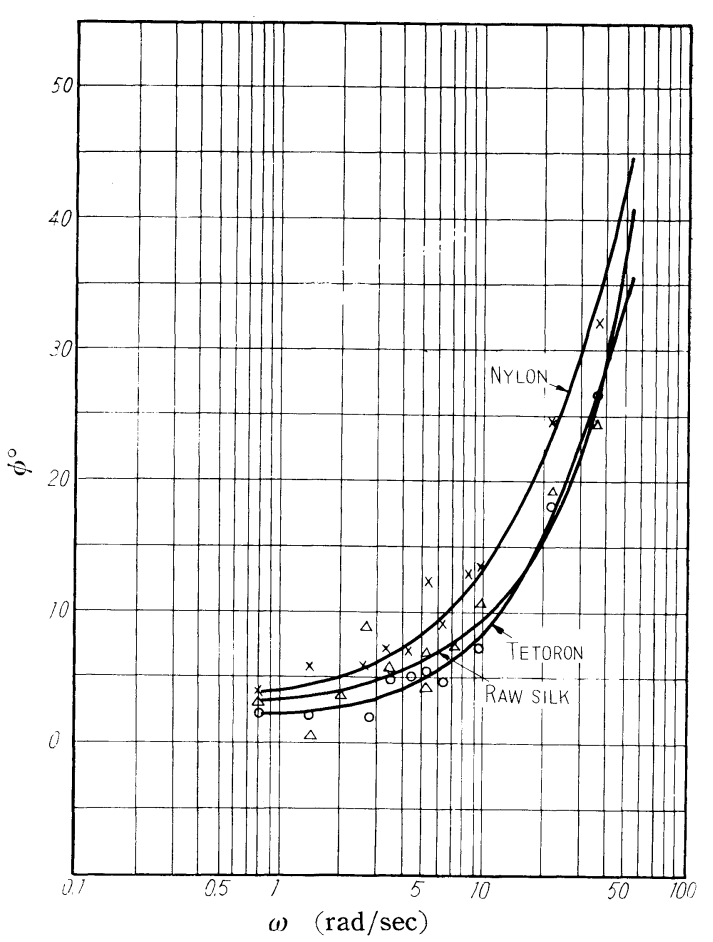

Fig. 34 Bode diagram (phase angle) of fiber tested under extension input

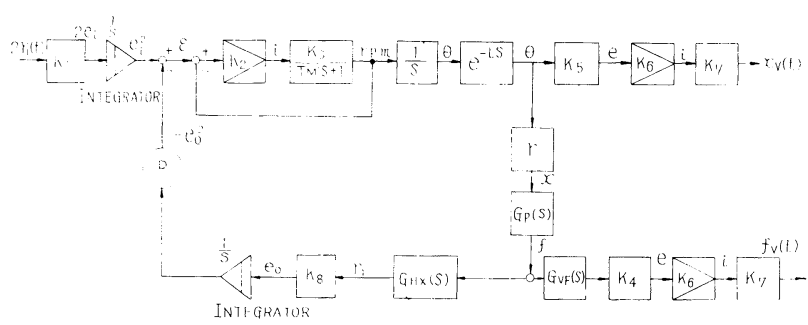

Fig. 35 Block diagram of tester of load-extension behevior with any sectional area of test piece taken as input signal

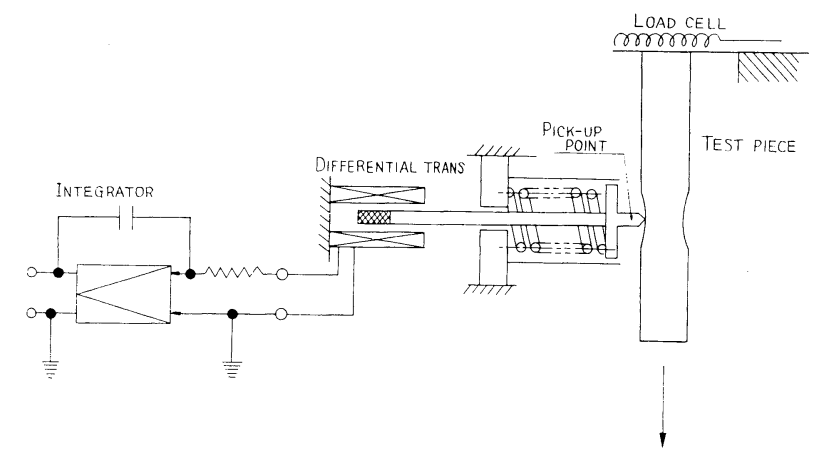

Fig. 36 Detector of sectional area of test piece 
hand to balance the two voltages. Therefore, the servo-motor must be driven until the difference between the two voltages is zero, so that a sectional area which conforms with a pre-set program can be taken as input.

Extensions during a test like this convert the rotation angle into voltage by means of the rotary potentiometer connected to the servo-motor. They are then transmitted to the recorder through the D.C. amplifier. The force generated in the test piece is detected by the strain meter through block elements $G_{p}(s)$ and $G_{V F}(s)$. Then it is transmitted into the recorder, as the extensions are. The overall transfer function of the tester is obtainable from Fig. 36 as follows :

$$
G_{o}(s)=\frac{K_{a} G_{p}(s) G_{H X}(s)}{s^{2}\left(T_{M} s+1\right)} e^{-L s}
$$

where $K_{a}=K_{M} K_{8} r P$

Therefore, the open-loop frequency response of the tester is:

$$
\begin{aligned}
& \left|G_{o}\right|=K_{a}\left|G_{p}(j \omega)\right| G_{H X}(j(\omega)) \\
& \omega^{2} \sqrt{ } 1+\left(T_{M \omega}\right)^{2} \\
& N \mathrm{~dB}=20 \log _{10} K_{a}+20 \log _{10} G_{p}(j \omega) \\
& +20 \log _{10} G_{H X}(j \omega)-40 \log _{10}(\omega) \\
& -10 \log _{10}\left\{1+\left(T_{M \omega}\right)^{2}\right\} \\
& \phi=\angle G_{p}(j \omega)+\angle G_{H X}(j \omega)-\angle 180^{\circ}-\tan { }^{1} T_{M \omega} \\
& -\angle L \omega) \quad \cdots \cdots \cdots(65)
\end{aligned}
$$

Take two closed-loop transfer functions: $G_{V x}(s)$ where the output is extension; and $G_{v f}(s)$ where the ontput is force (a vertical component load). The for. mer is

$$
\begin{aligned}
& G_{V x}(s)=\begin{array}{l}
X_{V}(s) \\
A(s)
\end{array} \\
& =T_{M} s^{3}+s^{2}-\left\{K_{a} L G_{p}(s) G_{H X}(s)\right\} s+K_{a} G_{p}(s) G_{H X}(s) e^{L s} \\
& \quad \cdots \cdots \cdots \cdots 66
\end{aligned}
$$

where $X_{V}(s)$ is the Laplace transform of the vertical component strain; $A(s)$ is the sectional area of the test piece ;

$$
K_{b}=K_{1} K_{5} K_{6} K_{7} K_{M}
$$

and $e^{-L S}$ in the denominator is approximated by (1$L s)$, as previously done.

With eq. (66) taken as the following general for. mula, the closed-loop frequency response is expressible as follows:

$$
\begin{aligned}
& G_{V x}(s)=\frac{K_{b}}{a_{1} s^{3}+b_{1} s^{2}-c_{1} s+d_{1}} e^{-L S} \quad \cdots \cdots \cdots(67) \\
& \left|G_{V x}\right|=\frac{K_{b}}{\sqrt{\left(d_{1}-b_{1} \omega^{2}\right)^{2}}+\left(\overline{a_{1} \omega^{2}}+c_{1}\right)^{2} \omega^{2}} \cdots \cdots \cdots(68) \\
& N \mathrm{~dB}=20 \log _{10} K_{b}-10 \log _{10}\left\{\left(d_{1}-b_{1} \omega^{2}\right)^{2}+\left(a_{1 \omega}\right)^{2}\right. \\
& \left.\left.+c_{1}\right)^{2} \omega^{2}\right\} \quad \ldots \ldots \ldots .699 \\
& \phi=-\tan \begin{array}{c}
{ }_{1}\left(a_{1} \omega^{2}+c_{1}\right) \omega \\
d_{1}-b_{1} \omega^{2}
\end{array}-\angle L \omega
\end{aligned}
$$

and the latter is :

$$
\begin{aligned}
& G_{V f}(s)=\frac{F_{V}(s)}{A(s)} \\
& ={ }^{K_{c} G_{p}(s) G_{V F}(s)} \\
& T_{M} s^{3}+s^{2}-\left\{K_{a} L G_{p}(s) G_{H X}(s)\right\} s+K_{a} G_{p}(s) G_{H X}(s)^{e^{-L S}} \\
& \cdots \cdots \cdots \cdots(71)
\end{aligned}
$$

where

$$
K_{c}=K_{1} K_{M} K_{4} K_{6} K_{7} r
$$

With eq. (71) taken as the following general formula, we get the closed-loop frequency as follows:

$$
\begin{gathered}
G_{V f}(s)=\frac{K_{c} G_{p}(s) G_{V F}(s)}{a_{2} s^{3}+b_{2} s^{2}-c_{2} s+d_{2}} e-{ }^{L S} \cdots \cdots \cdots(72) \\
G_{V f} \mid=\frac{K_{c}\left|G_{p}(j \omega)\right| G_{V F}(j \omega)}{\sqrt{ }\left(d_{2}-b_{2} \omega^{2}\right)^{2}+\left(a_{2} \omega^{2}+c_{2}\right)^{2} \omega^{2}} \cdots \cdots \cdots(73) \\
N \mathrm{~dB}=20 \log _{10} K_{c}+20 \log _{10} \mid G_{p}(j \omega) \\
\quad+20 \log _{10} \mid G_{V F}(j \omega)-10 \log _{10}\left\{\left(d_{2}-b_{2} \omega^{2}\right)^{2}\right. \\
\left.\quad+\left(a_{2} \omega^{2}+c_{2}\right)^{2} \omega^{2}\right\} \\
\cdots=\angle G_{p}(j \omega)+\angle G_{V F}(j \omega)-\tan ^{-1}\left(a_{2} \omega^{2}+c_{2}\right) \omega \\
d_{2}-b_{2} \omega^{2} \\
-\angle L \omega
\end{gathered}
$$

5-2. Method of Testing with the Horizontal Component Load of Test Piece Taken as Input

It is considered a matter of course that a material differs in characteristics depending on whether we leave its entire horizontal component strain alone, as is done in a usual tension test, or restrict it. With this in mind, we will discuss a method of tension test in which the horizontal load is increased at a constant speed. Fig. 37 shows the detector of the horizontal component load of the test piece.

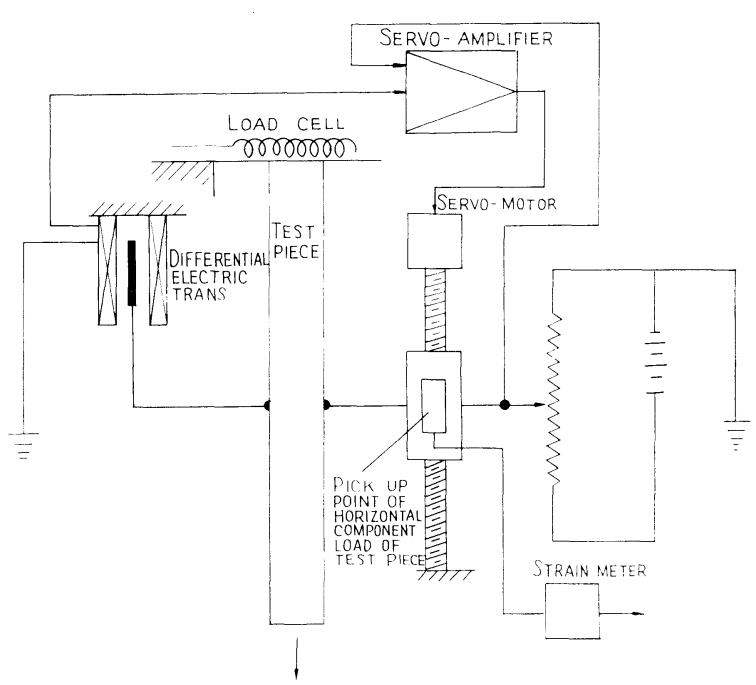

Fig. 37 Detector of horizontal component load of test piece and position control system of pickup point 
First, we fix a load pick up point on one side of the middle of the test piece; and a position pickup point on the other side. The position pickup point causes the load pickup point to follow the vertical strain during a tension test. When the position pick up point is shifted by the vertical strain of the test piece during a test, the displacement is transformed into voltage by the differential electric transformer, and then compared with the voltage from the potentiometer which is connected to the transmitter. Thus we can make a test with the relative positions of the two pickup points, on the one hand, and the test piece, on the other, kept stable. Fig. 38 is a block diagram of this tester:

where

$K_{4}^{\prime}$ : gain constant of strain meter

$G_{H F}(s)$ : transfer function of material by which force generated in test piece is convert. ed into horizontal component load.

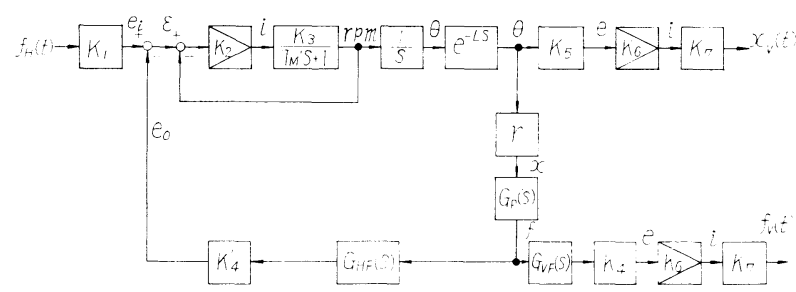

Fig. 38 Block diagram of tester of load-extension behavior with any horizontal component load of test piece taken as input signal

The input voltage of a horizontal component load programmed in the analog computer is transmitted to the servo-motor, as described earlier, and gives vertical component stain to the test piece by driving the extension terminal connected to the test piece.

The horizontal component strain generated in a test piece is controlled by the two pickup points. This is tantamount to imposing a horizontal component load. The horizontal component load is converted into voltage by the strain meter, and then compared with input voltage. Thus the servo-motor is driven until the differential voltage is zero, so that the horizontal component load is put accurately on the test piece. At the same time, the vertical component strain of the test piece, as well as its vertical component load, is detected and recorded.

The overall transfer function of the tester is obtainable from Fig. 38 as follows:

$$
G_{0}(s)=\frac{K_{d} G_{p}(s) G_{H F}(s)}{s\left(T_{M} s+1\right)} e^{L S}
$$

where $K_{d}=K_{M} K_{4}{ }^{\prime} r$

Therefore, the closed-loop frequency response is :

$$
\begin{aligned}
\left|G_{0}\right| & =\frac{K_{d}\left|G_{p}(j \omega)\right| G_{H F}(j \omega)}{\omega \sqrt{ } 1+\left(T_{M \omega}\right)^{2}} \\
N \mathrm{bB} & =20 \log _{10} K_{d}+20 \log _{10} \mid G_{p}(j \omega) \\
& +20 \log _{10}\left|G_{H F}(j \omega)\right|-20 \log _{10} \omega \\
& \quad-10 \log _{10}\left\{1+\left(T_{M \omega}\right)^{2}\right\} \\
\phi= & \angle G_{p}(j \omega)+\angle G_{H F}(j \omega)-\angle 90^{\circ} \\
- & \tan ^{-1} T_{M \omega}-\angle L \omega
\end{aligned}
$$

The closed-loop transfer function differs depend. ing on whether output or force (vertical component load) is taken as extension. The function in the former case is :

$$
\begin{gathered}
G_{V x}(s)=\frac{X_{V}(\mathrm{~s})}{F_{H}(s)} \\
=\frac{K_{e}}{T_{M} s^{2}+\left\{1-K_{d} L G_{p}(s) G_{H F}(s)\right\} s+K_{d} G_{p}(s) G_{H F}(s)^{e^{-L s}}}
\end{gathered}
$$

where $F_{H}(s)$ is the Laplace transformation of the horizontal component load, and $K_{e}=K_{1} K_{M} K_{5} K_{6} K_{7}$

With Eq. (80) expressed by the following general formula, the closed-loop frequency response is:

$$
\begin{aligned}
& \left.G_{V x}(s)=\frac{K_{e}}{a_{3} s^{2}+b_{3} s+c_{3}} e^{-L s} \quad \ldots \ldots \ldots \cdot s 1\right) \\
& G_{V x}=\frac{K_{e}}{\sqrt{ }\left(c_{3}-a_{3} \omega^{2}\right)^{2}+\left(b_{3} \omega\right)^{2}} \\
& N \mathrm{~dB}=20 \log _{10} K_{e}-10 \log _{10}\left\{\left(c_{3}-a_{3}\left(\omega^{2}\right)^{2}+\left(b_{3} \omega\right)^{2}\right\}\right. \\
& \left.\phi=-\tan ^{-1} b_{3}-a_{3} \omega\right)^{2}-\angle L \omega
\end{aligned}
$$

The transfer function with force taken as extension is :

$$
\begin{aligned}
& G_{V f}(s)=\begin{array}{l}
F_{V}(s) \\
F_{H}(s)
\end{array} \\
= & K_{f} G_{p}(s) G_{V F}(s) \\
= & T_{M} s^{2}+\left\{1-K_{d} G_{p}(s) G_{H F}(s)\right\} s+K_{d} G_{p}(s) G_{H F}(s)^{e^{-L S}}
\end{aligned}
$$

where $F_{V}(s)$ is the Laplace transform of the vertical component load, and $K_{f}=K_{1} K_{M} K_{4} K_{6} K_{7} r$

With eq. (85) taken as the following general formula, we get the closed-loop frequency response as follows :

$$
\begin{aligned}
G_{V f}(s) & =\begin{array}{l}
K_{f} G_{p}(s) G_{V F}(s) \\
a_{4} s^{2}+b_{4} s+c_{4}
\end{array} e^{-L S} \\
G_{V f} \mid & =\frac{K_{f}\left|G_{p}(j \omega)\right| G_{V F}(j \omega)}{\sqrt{\left(c_{4}-a_{4} \omega^{2}\right)^{2}+\left(b_{4} \omega\right)^{2}}} \\
N \mathrm{~dB} & =20 \log _{10} K_{f}+20 \log _{10} \mid G_{p}(j \omega) \\
& +20 \log _{10} G_{V F}(j \omega)-10 \log _{10}\left\{\left(c_{4}-a_{4} \omega^{2}\right)^{2}\right. \\
& \left.+\left(b_{4} \omega\right)^{2}\right\} \\
\phi= & \ldots G_{p}(j \omega)+\angle G_{V F}(j \omega)-\tan ^{-1} \frac{b_{4} \omega}{\left.c_{4}-a_{4} \omega\right)^{2}} \\
- & \angle L \omega \cdots(86)
\end{aligned}
$$


5-3 Method of Testing with Stress of Test Piece Taken as Input

To obtain a stress strain curve from a load exten sion curve by using the conventional tension tester, we must keep correcting genuine stress as the sectional area of the test piece changes during a test, because stress is often shown by the apparent stress. To remove this inconvenience, a method of testing with the stress of a test piece taken as input is needed. Fig. 39 is a signal-flow diagram of our tester of stress input.

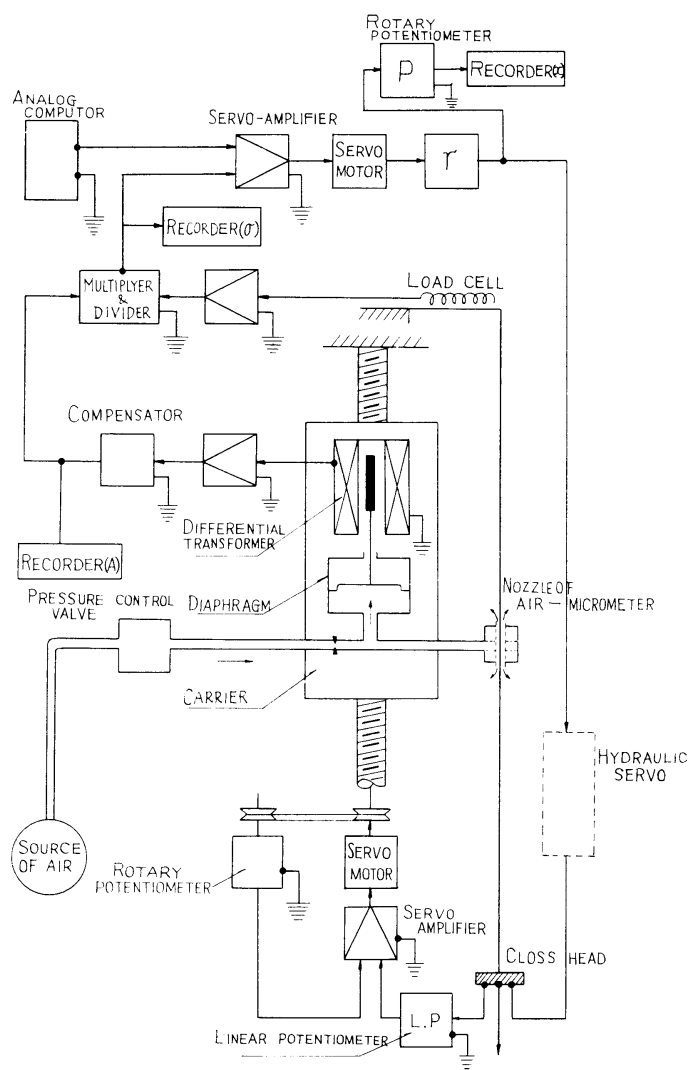

Fig. 39 Signal-flow diagram of tester of load-extension behavior with any stress of test piece taken as input signal

As this figure shows, the sectional area of a test piece is detected by an air-micrometer. Its back pressure drives the diaphragm valve. Its displacement is converted into voltage by the differential transformer. This voltage is adjusted to a voltage which is proportional to the sectional area by the compensator.

The load imposed on a test piece is detected by the load cell and divided by the voltage which is proportional to the above-mentioned sectional area. Thus we can feedback the load in the form of the so-called genuine stress. Stress of an arbitrary function is loaded faithfully on the test piece and its displacement output recorded.

A block diagram showing stress used as input is given in Fig, 40.

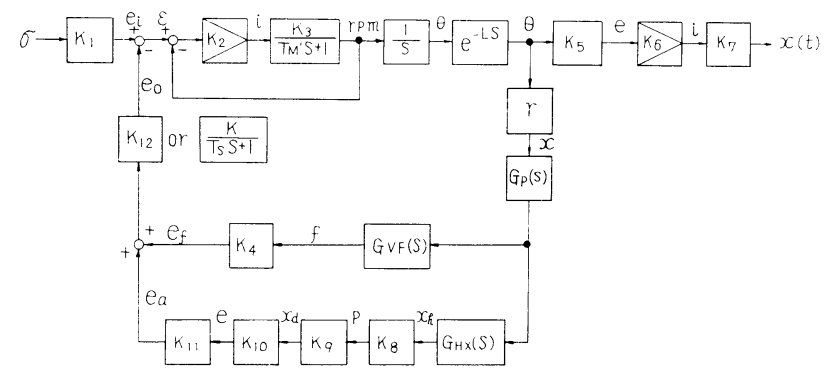

Fig. 40 Block diagram of tester of load extension behavior with any stress of test piece taken as input signal

The overall transfer function is obtained from this figure as follows :

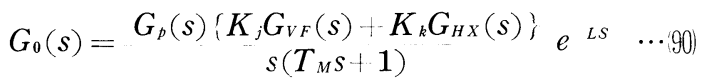

where $K_{j}=K_{M} K_{4} K_{12} r$

$$
\text { and } \quad K_{k}=K_{M} K_{8} K_{9} K_{10} K_{11} K_{12} r
$$

Therefore, the open-loop frequency response is:

$$
\begin{aligned}
& \begin{array}{r}
G_{0}=G_{p}(j(1)):\left\{K_{j} G_{V F}(j())+K_{k} G_{H X}(j(\omega))\right\} \\
\omega \sqrt{ } \mathbf{1}+\left(T_{M}(\omega)^{2}\right.
\end{array} \\
& N \mathrm{~dB}=20 \log _{10} G_{p}(j \omega)+20 \log _{10} \mid\left\{K_{j} G_{V F}(j \omega)\right) \\
& \left.+K_{k} G_{H X}(j \omega)\right\} \mid-20 \log _{10 \omega}-10 \log _{10}\{1 \\
& \left.+\left(T_{M \omega}\right)^{2}\right\} \quad \text { …....929) } \\
& \phi=\angle G_{p}(j \omega)+\angle\left\{K_{j} G_{V F}(j \omega)+K_{k} G_{H X}(j \omega)\right\} \\
& \left.-\angle 90^{\circ}-\tan { }^{\circ} T_{M(\omega)}-\angle L \omega\right)
\end{aligned}
$$

The closed loop transfer function differs, depending on whether we use, for multiplication and division, an separate electronic instruments $\left(K_{12}\right)$ or a servomultiplier and an amplifier $\left[K /\left(T_{M} S+1\right)\right]$

When we use electronic-type instruments

$$
\begin{gathered}
G(s)=K_{e ?}{ }^{-L S} \\
T_{M} s^{2}+\left[1-L G_{p}(s)\left\{K_{j} G_{V F}(s)\right.\right. \\
\left.\left.\left.\left.+K_{k} G_{H X}(s)\right\}\right] s+G_{p}(s)\right\} K_{j} G_{V F}^{-}(s)+K_{k} G_{H X}(s)\right\}
\end{gathered}
$$

where $K_{e}=K_{M} K_{1} K_{5} K_{6} K_{7}$

Therefore, frequency response, expressed by the general formula given earlier, is :

$$
\begin{aligned}
& G(s)=\frac{K_{l}}{a_{5} j^{2}+b_{5} s+c_{5}} e^{-L S} \\
& |G|=\frac{K_{l}}{\left.\sqrt{ }\left(c_{5}-a_{5} \omega\right)^{2}\right)^{2}+\left(b_{5} \omega\right)^{2}}
\end{aligned}
$$




$$
\begin{aligned}
& \left.N \mathrm{~dB}=20 \log _{10} K_{l}-10 \log _{10}\left(c_{5}-a_{5} \omega^{2}\right)^{2}+\left(b_{5} \omega\right)^{2}\right\} \\
& \ldots \ldots \ldots \ldots(97) \\
& \phi=-\tan ^{-1} \frac{b_{5} \omega}{c_{5}-a_{5} \omega^{2}}-\angle L \omega
\end{aligned}
$$

\section{5-4 Method of Measuring Poisson's Ratio}

Poisson's ratio can be measured by measuring the vertical and horizontal component strains of a test piece under prescribed load input by our tester and using multiplication and division instruments together. The details are omitted.

\section{Conclusions}

We have investigated and analyzed the characteristics and practical utility of an all-purpose, electronic tensile tester experimentally produced.

Experiments have shown that tests under load or extension input, e.q., repeated stress tests and constant. speed load input tests-hitherto difficult - can be made easily by our tester. As we have shown by examples of applied tests, our tester is practical and useful for tests of industrial materials.

\section{References :}

[1] F. Murakami ; Process analysis for textile engineers, 17, RIK Japan (1964)

[2] S. Watanabe ; Joxrnal of the Textile Machinery Society of Japan, Transactions, 12, 163 (1959)

[3] M. Terao and T. Nakagawa ; Self-balancing type instrument, OHM, Japan (1964)

[4] K. Izawa; Introduction to Automatic Control, ELSEVIER (1963)

[5] Y. Takahashi; Control system design notes, KYC̄ RITSU, Japan (1963)

[6] D. Graham and D. McRuer; Analysis of nonlinear control system, JOHN WILEY \& SONS, INC. (1961)

[7] Floyd. E. Nixon; Handbook of Laplace transformation, PRENTICE-HALL (1965)

[8] G. Doetsch ; Anleitung zum praktischen gebrauch der Laplace-Transformation, R. OLDENBOURG VERLAG, MÜNCHEN. (1956) 